\title{
PSEUDO-BOUNDARIES AND PSEUDO-INTERIORS IN EUCLIDEAN SPACES AND TOPOLOGICAL MANIFOLDS
}

\author{
BY \\ ROSS GEOGHEGAN( ${ }^{1}$ ) AND R. RICHARD SUMMERHILL
}

\begin{abstract}
The negligibility theorems of infinite-dimensional topology have finitedimensional analogues. The role of the Hilbert cube $I^{\omega}$ is played by euclidean $n$-space $E^{n}$, and for any nonnegative integer $k<n, k$-dimensional dense $F_{\sigma}$-subsets of $E^{n}$ exist which play the role of the pseudo-boundary of $I^{\omega}$. Their complements are $(n-k-1)$-dimensional dense $G_{\delta}$ pseudo-interiors of $E^{n}$. Two kinds of $k$-dimensional pseudo-boundaries are constructed, one from universal compacta, the other from polyhedra. All the constructions extend to topological manifolds.
\end{abstract}

1. Introduction. In this paper we use the methods of infinite-dimensional topology to derive new information about the point-set topology of euclidean spaces and topological manifolds. We hope that this new information will turn out to be useful, but we also hope that by presenting infinite-dimensional ideas in a familiar finite-dimensional context, we will make them more accessible.

The idea is to partition euclidean $n$-space $E^{n}$ into a $k$-dimensional pseudoboundary $(-1 \leq k<n)$ and an $(n-k-1)$-dimensional pseudo-interior, and to derive negligibility theorems analogous to those of infinite-dimensional topology.

We now give five typical theorems and point out some analogies. First, a definition: A subset $X$ of a metric space $Y$ (whose metric is $d$ ) is strongly negligible in $Y$ if for each open set $U$ in $Y$ and each continuous function $\varepsilon: U \rightarrow \mathbf{R}^{+}$, there is a homeomorphism $h: Y \rightarrow Y-(X \cap U)$ such that $h$ fixes $Y-U$ and $d(x, h(x))<\varepsilon(x)$ for each $x \in U$. Note: Strong negligibility is a topological property independent of the metric $d$.

Theorem 1.1. Let $k$ and $n$ be integers with $0 \leq k<n$. $E^{n}$ is the union of two disjoint dense subsets $B^{k}$ (of dimension $k$ ) and $P^{n-k-1}$ (of dimension $\left.n-k-1\right)$ such that

(1) if $n \leq 2 k+1$, then any $\sigma$-compact subset of $P^{n-k-1}$ is strongly negligible in $P^{n-k-1}$, and

(2) if $n \geq 2 k+1$, then any compact subset of $B^{k}$ is strongly negligible in $B^{k}$.

Theorem 1.2. Let $k \geq 0$ be an integer. $E^{2 k+1}$ is the union of two disjoint dense $k$ dimensional subsets $P^{k}$ and $B^{k}$ such that

Presented to the Society, April 26, 1972; received by the editors June 27, 1972 and, in revised form, May 10, 1973.

AMS (MOS) subject classifications (1970). Primary 57A15; Secondary 57A20.

(1) Supported by National Science Foundation grant GP-7952X3.

Copyright $O$ 1974, American Mathematical Society 
(1) any $\sigma$-compact subset of $P^{k}$ is strongly negligible in $P^{k}$,

(2) any compact subset of $B^{k}$ is strongly negligible in $B^{k}$, and

(3) any compact metric space of dimension $\leq k$ can be embedded in $B^{k}$ or in $P^{k}$.

The analogy with the infinite-dimensional case is best understood by reading Theorem 1.2 with $I^{\omega}$ replacing $E^{2 k+1}, k$ being infinite. Then $B$ and $P$ can be taken to be the usual pseudo-boundary and pseudo-interior, respectively, of $I^{\omega}$.

The space $B^{k}$ of 1.1 and 1.2 is the universal $k$-dimensional pseudo-boundary of $E^{n}$, which is defined and studied in $\$ 3$.

Another kind of $k$-dimensional pseudo-boundary of $E^{n}$ can be built out of $k$ dimensional polyhedra. It is easily described: Let $J_{0}$ be a rectilinear PL triangulation of $E^{n}$ all of whose $n$-simplexes have the same diameter. For each integer $i \geq 1$, let $J_{i}$ be the $i$ th barycentric subdivision of $J_{0}$ and let $J_{i}^{k}$ be the $k$ skeleton of $J_{i}$. The polyhedral $k$-dimensional pseudo-boundary of $E^{n}$ is $\tilde{B}_{n}^{k}$ $=\cup_{i=1}^{\infty}\left|J_{i}^{k}\right|$. The corresponding $(n-k-1)$-dimensional pseudo-interior is $\tilde{P}_{n}^{n-k-1}=E^{n}-\tilde{B}_{n}^{k}$.

In the theorems which follow, as in the paper generally, a space $X$ is a polyhedron if there is a countable locally finite simplicial complex $K$ such that $X$ is homeomorphic to $|K|$; a subpolyhedron of $E^{n}$ is the body of such a complex when linearly embedded as a closed subset in $E^{n}$; a polyhedron in $E^{n}$ is tame if there is a homeomorphism $h$ of $E^{n}$ such that $h(X)$ is a subpolyhedron of $E^{n}$.

Here are some theorems which illustrate the properties of the polyhedral pseudo-boundaries.

Theorem 1.3. Let $k$ and $n$ be integers, $-1 \leq k<n$.

(1) Any subpolyhedron of $E^{n}$ whose dimension $\leq n-k-1$ can be embedded in $\tilde{P}_{n}^{n-k-1}$ so as to be tame in $E^{n}$.

(2) If $n \leq 2 k+1$ and $n \neq 4$, then the countable union of polyhedra, each tame in $E^{n}$ and lying in $\tilde{P}_{n}^{n-k-1}$, is strongly negligible in $\tilde{P}_{n}^{n-k-1}$.

(3) If $n \geq 2 k+1$ and $n \neq 4$, then any compact subset of $\tilde{B}_{n}^{k}$ is strongly negligible in $\tilde{B}_{n}^{k}$.

Theorem 1.4. Let $k$ and $n$ be integers, $-1 \leq k \leq n-3, k \neq 1, n \neq 4$. The countable union of compact (topological) polyhedra of dimension $\leq k$ in $\tilde{P}_{n}^{n-k-1}$ is strongly negligible in $\tilde{P}_{n}^{n-k-1}$.

Theorem 1.5. Let $k \geq 0$ be an integer.

(1) If $k \neq 1$, then the countable union of compact (topological) polyhedra in $\tilde{P}_{2 k+1}^{k}$ is strongly negligible in $\tilde{P}_{2 k+1}^{k}$.

(2) Any compact subset of $\tilde{B}_{2 k+1}^{k}$ is strongly negligible in $\tilde{B}_{2 k+1}^{k}$.

(3) Any compact polyhedron of dimension $\leq k$ can be embedded in $\tilde{B}_{2 k+1}^{k}$ or in $\tilde{P}_{2 k+1}^{k}$.

Again, a close analogy with the infinite-dimensional case is discovered by reading Theorem 1.5 with $I^{\omega}$ replacing $E^{2 k+1}, k$ being infinite. But since this 
requires some knowledge of Anderson's f.d. cap sets, we postpone further discussion of the analogy until the introduction to $\$ 4$.

Theorems 1.1 through 1.5 are not our only negligibility theorems. For example, in Theorem 3.20 we completely characterize the strongly negligible subsets of the universal pseudo-interiors of $E^{n}$ by an elementary homotopy condition analogous to Anderson's Property Z. In Theorem 3.24 we do something similar in the pseudo-boundary.

The methods used in this paper have many sources in the literature. We have chosen to discuss these in the introductions to $\S \S 2,3$, and 4 rather than here. The ideas from infinite-dimensional topology are all in $\$ 2$. These are applied to $E^{n}$ in $\S 3$ and $\S 4$. The extension to topological manifolds is in $\S 5$. Theorems 1.1 and 1.2 are proved at the end of $\S 3$. Theorems $1.3,1.4$, and 1.5 are proved at the end of $\S 4$.

This paper contains the proofs of all theorems claimed in [16].

Added in proof. A further analogy between the finite-dimensional and infinitedimensional pseudo-interiors is given in [33].

Notation and terminology. $\mathbf{R}$ denotes the real numbers, $\mathbf{R}^{+}$the positive real numbers. $E^{n}$ is the $n$-fold product of R. All metrics are denoted by $d$. In part B of $\S 3, E^{n}$ carries the maximum metric; elsewhere the choice of complete metric on $E^{n}$ is immaterial. A continuous function is called a map. The identity map of $Y$ is denoted by $1_{Y}$ or simply 1 . The restriction of a map $f$ to a subset $X$ of its domain is denoted by $f \mid X . Y$ is $\sigma$-compact if it is the countable union of compact spaces. A compactum is a compact metric space. The terms strongly negligible, polyhedron, subpolyhedron, and tame have already been defined. Unless otherwise stated, an open subset of $E^{n}$ carries the PL structure inherited from the standard PL structure of $E^{n}$.

2. Pseudo-boundaries and pseudo-interiors in complete metric spaces. In this section we give a short account of the basic point-set topology which underlies the negligibility theory of infinite-dimensional manifolds. Our context is: given a complete metric space $Y$, when can $Y$ be partitioned into a set $B$ (analogous to the pseudo-boundary of the Hilbert cube $I^{\omega}$ ) and a set $P$ (analogous to the pseudo-interior of $I^{\omega}$ ) so that "tame" subsets of $B$ or $P$ are negligible? And what constitutes a family of "tame" sets? We begin with a short history, no knowledge of which is needed in order to understand the section.

The first attempts to axiomatize such ideas occurred simultaneously in R.D. Anderson's paper [2] and in Bessaga-Pełczyński's paper [4]. Their aim was the classification up to homeomorphism of certain kinds of incomplete metrizable linear spaces. Anderson's (f.d.) cap sets and Bessaga-Pełczyński's homogeneous collections and skeletons give rise to special cases of what we call a "pseudoboundary."

The next generalization was to study the case where $Y$ is a Hilbert manifold 
(or Hilbert cube manifold) and the pseudo-boundaries are dense submanifolds modelled on incomplete linear spaces. This was done by West [32] (whose results were applied by Henderson-West in [18]) and by Chapman [12], [13], [14].

That an arbitrary complete metric space (rather than an infinite-dimensional manifold) is the correct setting, was recognized simultaneously in the paper of West [32] mentioned above, and in Torunczyk's paper [31]. In fact, we must acknowledge that many of the theorems in this section are implicitly present in those papers. Nevertheless, we have felt it necessary to include the section for a number of reasons. Our definition of pseudo-boundary corresponds to West's and is different from Torunczyk's: the difference is unimportant in the usual infinite-dimensional applications but is critical in $E^{n}$. Yet we cannot simply quote West because he only proves the basic uniqueness theorem ( 2.5 in this paper), while we want the negligibility theorems. Again, even allowing for the different definitions, Torunczyk uses more axioms than are nesessary in proving his negligibility theorems.

The ideas in this chapter are quite elementary. The main theorems are 2.5, 2.8, 2.13, and 2.16. Lemma 2.21 will also be useful in the subsequent sections.

Throughout $\S 2, Y$ will be a complete metric space with metric $d$.

A. Pseudo-boundaries and the axioms. Let $H(Y)$ be the set of homeomorphisms of the complete metric space $Y$. If $U$ is an open subset of $Y, \varepsilon: U \rightarrow \mathbf{R}^{+}$is a map, and $f \in H(Y)$, let

$$
\begin{aligned}
& V_{U}(f, \varepsilon)=\left\{h \in H(Y) \mid d(f(y), h(y))+d\left(f^{-1}(y), h^{-1}(y)\right)<\varepsilon(y)\right. \\
& \quad \text { for each } y \in Y \text {, and } h|Y-U=f| Y-U\} .
\end{aligned}
$$

Lemma 2.1. Let $U$ be an open subset of $Y$, let $\varepsilon: U \rightarrow(0,1]$ be a map, and let $\left\{f_{i}\right\}$ be a sequence of homeomorphisms of $Y$ such that $f_{i+1} \in V_{U}\left(f_{i}, \varepsilon / 2^{i}\right)$ for each $i \geq 1$. Then $f=\lim _{i \rightarrow \infty} f_{i}$ exists and lies in $V_{U}\left(f_{1}, \varepsilon\right)$.

Proof. $\left\{f_{i}\right\}$ and $\left\{f_{i}^{-1}\right\}$ are uniformly cauchy.

A subset $X$ of $Y$ is said to be thin in $Y$ if for each open subset $U$ of $Y$ containing $X$, and each map $\varepsilon: U \rightarrow \mathbf{R}^{+}$, there exists $h \in V_{U}(1, \varepsilon)$ such that $h(X) \cap X=\varnothing$.

Now let $\delta$ be some collection of subsets of $Y$. Let $\delta_{+}$[resp. $\delta_{++}$] be the collection of all finite [resp. countable] unions of closed subsets of elements of $\delta$.

A subset $B$ of $Y$ is called a pseudo-boundary for $\mathcal{S}$ in $Y$ if $B \in \mathcal{S}_{++}$and the following absorption property holds:

(*) for each $S \in \delta$, each open set $U$ in $Y$, and each map $\varepsilon: U \rightarrow \mathbf{R}^{+}$, there exists $h \in V_{U}(1, \varepsilon)$ such that $h(S \cap U) \subset B$.

If $B$ is a pseudo-boundary for $\mathcal{S}$ in $Y$, then $P=Y-B$ is called a pseudointerior for $\mathcal{S}$ in $Y$.

At various times we will assume that $\delta$ satisfies one or more of three axioms which we now state:

Axiom 1 (closed). Each element of $\delta$ is closed in $Y$. 
Axiom 2 (invariant). If $S \in \delta$ and $h \in H(Y)$, then $h(S) \in \delta$.

Axiom 3 (thin). Each element of $\delta_{+}$is thin in $Y$.

Lemma 2.2. Let $U$ be an open subset of $Y$, let $\varepsilon: U \rightarrow \mathbf{R}^{+}$be a map, and let $g \in H(Y)$. Then there exists a map $\delta: g(U) \rightarrow \mathbf{R}^{+}$such that $f g \in V_{U}(g, \varepsilon)$ and $g^{-1} f g \in V_{U}(1, \varepsilon)$ whenever $f \in V_{g(U)}(1, \delta)$.

Proof. Tedious, elementary, left to reader.

Two remarks follow immediately from 2.2 .

Remark 2.3. If $\delta$ satisfies Axiom 2, then the absorption property (*) is equivalent to

(**) for each subset $S$ of an element $\delta$, each open subset $U$ of $Y$, each map $\varepsilon: U \rightarrow \mathbf{R}^{+}$, and each $g \in H(Y)$, there exists $h \in V_{U}(g, \varepsilon)$ such that $h(S \cap U)$ $\subset B$.

Remark 2.4. If $\delta$ satisfies Axiom $2, B$ is a pseudo-boundary for $\delta$ in $Y$, and $g \in H(Y)$, then $g(B)$ is a pseudo-boundary for $\delta$ in $Y$.

B. Uniqueness of pseudo-boundaries. We prove here that whenever $\delta$ satisfies Axioms 1 and 2, pseudo-boundaries for $\delta$ in $Y$ are unique up to homeomorphism of $Y$.

Theorem 2.5 [32]. Let $\delta$ satisfy Axioms 1 and 2. If $B$ and $B^{\prime}$ are pseudoboundaries for $\mathcal{S}$ in $Y$, then there exists $f \in H(Y)$ such that $f(B)=B^{\prime}$. In fact, if $U$ is an open subset of $Y$ and $\epsilon: U \rightarrow \mathbf{R}^{+}$is a map, then there exists $f \in V_{U}(1, \epsilon)$ such that $f(B \cap U)=B^{\prime} \cap U$.

Proof. Let $B=\cup_{i=1}^{\infty} S_{i}$ and $B^{\prime}=\cup_{i=1}^{\infty} S_{i}^{\prime}$ where each $S_{i}$ and $S_{i}^{\prime}$ are closed subsets of elements of $\delta$. Assume $S_{1}=S_{1}^{\prime}=\varnothing$. We construct a sequence $\left\{f_{i}\right\}_{i \geq 1}$ in $H(Y)$ such that

(1) $f_{1}=1$ and $f_{i+1} \in V_{U}\left(f_{i}, \varepsilon / 2^{i}\right)$,

(2) $f_{i}\left(S_{i} \cap U\right) \subset B^{\prime}$ and $S_{i}^{\prime} \cap U \subset f_{i}(B)$, and

(3) $f_{i+1}=f_{i}$ on $S_{1} \cup \cdots \cup S_{i} \cup f_{i}^{-1}\left(S_{1}^{\prime} \cup \cdots \cup S_{i}^{\prime}\right)$ for each $i \geq 1$.

Then $f=\lim _{i \rightarrow \infty} f_{i}$ is the desired homeomorphism. Set $f_{1}=1$ and assume that $f_{1}, \ldots, f_{i}$ have been chosen. Let

$$
U^{\prime}=U-\left(S_{1} \cup \cdots \cup S_{i} \cup f_{i}^{-1}\left(S_{1}^{\prime} \cup \cdots \cup S_{i}^{\prime}\right)\right)
$$

Since $\mathcal{S}$ satisfies Axiom $1, U^{\prime}$ is open in $Y$. Now (Axiom 2 is used here!) apply (**) to $S_{i+1}, U^{\prime}, \varepsilon / 2^{i+1}, f_{i}$ and $B^{\prime}$ to obtain $h \in V_{U^{\prime}}\left(f_{i}, \varepsilon / 2^{i+1}\right)$ such that $h\left(S_{i+1} \cap U^{\prime}\right) \subset B^{\prime}$. Note that $h \in V_{U}\left(f_{i}, \varepsilon / 2^{i+1}\right), h=f_{i}$ on $S_{1} \cup \cdots \cup S_{i}$ $\cup f_{i}^{-1}\left(S_{1}^{\prime} \cup \cdots \cup S_{i}^{\prime}\right)$, and $h\left(S_{i+1} \cap U\right) \subset B^{\prime}$. Next let $U^{\prime \prime}=U-\left(h\left(S_{i+1}\right)\right.$ $\left.\cup S_{1}^{\prime} \cup \cdots \cup S_{i}^{\prime} \cup f_{i}\left(S_{1} \cup \cdots \cup S_{i}\right)\right)$ and apply (**) to $S_{i+1}^{\prime}, U^{\prime \prime}, \varepsilon / 2^{i+1}$, $h^{-1}$, and $B$ to obtain $g \in V_{U^{\prime \prime}}\left(h^{-1}, \varepsilon / 2^{i+1}\right)$ such that $g\left(S_{i+1}^{\prime} \cap U^{\prime \prime}\right) \subset B$. Then $g \in V_{U}\left(h^{-1}, \varepsilon / 2^{i+1}\right), g=h^{-1}$ on $h\left(S_{i+1}\right) \cup S_{1}^{\prime} \cup \cdots \cup S_{i}^{\prime} \cup f_{i}\left(S_{1} \cup \cdots \cup S_{i}\right)$, and $g\left(S_{i+1}^{\prime} \cap U\right) \subset B$. Then $f_{i+1}=g^{-1}$ is the homeomorphism which completes the induction.

2.2 and 2.5 give 
Corollary 2.6. Let $\delta$ satisfy Axioms 1 and 2 and let $B$ and $B^{\prime}$ be pseudo-boundaries for $\delta$ in $Y$. If $U$ is an open subset of $Y, \varepsilon: U \rightarrow \mathbf{R}^{+}$is a map, and $g \in H(Y)$, then there exists $f \in V_{U}(g, \varepsilon)$ such that $f(B \cap U)=B^{\prime} \cap g(U)$.

C. Negligibility from the pseudo-interior. Whenever $B$ is a pseudo-boundary for $\delta$ in $Y$ and $T \in \delta_{++}$, then $B \cup T$ is also a pseudo-boundary for $\delta$ in $Y$. This and Theorem 2.5 imply

Corollary 2.7. Let $\delta$ satisfy Axioms 1 and 2 and let $P$ be a pseudo-interior for $\delta$ in $Y$. If $T \in \mathcal{S}_{++}, U$ is an open subset of $Y$, and $\varepsilon: U \rightarrow \mathbf{R}^{+}$is a map, then there exists a homeomorphism $f \in V_{U}(1, \varepsilon)$ such that $f(P \cap U)=(P-T) \cap U$.

In our next corollary, we use the fact that for any subset $A$ of a metric space $B$, and for any map $\varepsilon: A \rightarrow \mathbf{R}^{+}$, there exists a map $\delta: B \rightarrow[0, \infty)$ such that $0<\delta(x)<\varepsilon(x)$ for each $x \in A$. We leave the proof of this elementary fact to the reader.

Corollary 2.8. Let $\delta$ satisfy Axioms 1 and 2 and let $P$ be a pseudo-interior for $\delta$ in $Y$. If $T \in \mathcal{S}_{++}$, then $P \cap T$ is strongly negligible in $P$.

Proof. Let $U$ be open in $P$, let $\varepsilon: U \rightarrow \mathbf{R}^{+}$be a map, and let $U^{\prime}$ be open in $Y$ with $U^{\prime} \cap P=U$. Then there is a mapping $\delta: Y \rightarrow[0, \infty)$ such that $0<\delta(x)$ $<\varepsilon(x)$ for each $x \in U$. Now $W=\delta^{-1}\left(\mathbf{R}^{+}\right) \cap U^{\prime}$ is an open subset of $Y$ and $\delta \mid W: W \rightarrow \mathbf{R}^{+}$is a map and hence, by Corollary 2.7, there exists a homeomorphism $f \in V_{W}(1, \delta)$ such that $f(P \cap W)=(P-T) \cap W$. Then $d(x, f(x))$ $<\delta(x)<\varepsilon(x)$ for each $x \in U, f$ fixes $Y-U$, and $f(P)=P-(P \cap T \cap U)$.

D. Negligibility from the pseudo-boundary. We prove here that elements of $\delta_{+}$ are negligible in the pseudo-boundary provided Axiom 3 holds.

Lemma 2.9. Let $\delta$ satisfy Axiom 3 and let $S, T \in \mathcal{S}_{+}$. If $U$ is an open subset of $Y$ containing $S \cap T$ and $\varepsilon: U \rightarrow \mathbf{R}^{+}$is a map, then there exists $h \in V_{U}(1, \varepsilon)$ such that $h(S) \cap T=\varnothing$.

Proof. Define $\delta: Y \rightarrow[0, \infty)$ by $\delta(x)=\frac{1}{2} d(x, Y-U)$ and let $R=\{x \in S \mid$ $d(x, T) \leq \delta(x)\} \cup\{x \in T \mid d(x, S) \leq \delta(x)\}$. Then $R \in \mathcal{S}_{+}$and hence is thin in $Y$ since $\delta$ satisfies Axiom 3. Now $U$ is open in $Y$ and contains $R$ and $\gamma: U \rightarrow \mathbf{R}^{+}$, defined by $\gamma(x)=\min \{\delta(x), \varepsilon(x)\}$ for each $x \in U$, is a map. Therefore there exists $h \in V_{U}(1, \gamma)$ such that $h(R) \cap R=\varnothing$. Then $h \in V_{U}(1, \varepsilon)$ and $h(S) \cap T=\varnothing$. A useful fact to recall here is that $d(x, h(x))<\delta(x)$ and $d\left(h^{-1}(h(x)), h(x)\right)=d(x, h(x))<\delta(h(x))$ for all $x$ in $U$.

2.2 and 2.9 give

Corollary 2.10. Let $\delta$ satisfy Axioms 2 and 3 and let $S, T \in \mathcal{S}_{+}$. Let $U$ be open in $Y$, let $\varepsilon: U \rightarrow \mathbf{R}^{+}$be a map, and let $g \in H(Y)$ be such that $g(S) \cap T \subset g(U)$. Then there exists $h \in V_{U}(g, \varepsilon)$ such that $h(S) \cap T=\varnothing$.

Lemma 2.11. Let $\delta$ satisfy Axioms 1,2 , and 3 , let $S, T \in \mathcal{S}_{++}$, let $U$ be open in 
$Y$, and let $\varepsilon: U \rightarrow \mathbf{R}^{+}$be a map. Then there exists $f \in V_{U}(1, \varepsilon)$ such that $f(S \cap U)$

$\cap T=\varnothing$.

Proof. Let $S=\cup_{i=1}^{\infty} S_{i}$ and $T=\cup_{i=1}^{\infty} T_{i}$ where $S_{1} \subset S_{2} \subset \cdots$ and $T_{1} \subset T_{2}$ $\subset \cdots$, and each $T_{i}, S_{i} \in \mathcal{S}_{+}$. We assume that $S_{1}=T_{1}=\varnothing$. For each $i \geq 1$, set $S_{i}^{\prime}=\left\{x \in S_{i} \mid d(x, Y-U) \geq 1 / i\right\}$ and $T_{i}^{\prime}=\left\{x \in T_{i} \mid d(x, Y-U) \geq 1 / i\right\}$. Then $S \cap U=\cup_{i=1}^{\infty} S_{i}^{\prime}$ and $T \cap U=\cup_{i=1}^{\infty} T_{i}^{\prime}$. We will construct a sequence $\left\{f_{i}\right\}_{i \geq 1}$ of homeomorphisms of $Y$ and a sequence $\left\{\delta_{i}\right\}_{i \geq 0}$ of maps from $U$ to $\mathbf{R}^{+}$ such that

(1) $f_{i}\left(S_{i}^{\prime}\right) \cap T_{i}^{\prime}=\varnothing$,

(2) $\delta_{i}(x)=d\left(f_{i}(x), T_{i}^{\prime}\right)$ for each $x \in S_{i}^{\prime}$, and

(3) $f_{1}=1$ and $f_{i+1} \in V_{U}\left(f_{i}, \gamma_{i} / 2^{i}\right)$ where $\gamma_{i}=\min \left\{\delta_{1}, \ldots, \delta_{i}, \varepsilon\right\}$ for each $i \geq 1$. Then $f=\lim _{i \rightarrow \infty} f_{i}$ is the desired homeomorphism, for the conditions imply that $f_{i}$ moves $S_{i}^{\prime}$ off $T_{i}^{\prime}$ and then $S_{i}^{\prime}$ is never again allowed to move far enough to hit $T_{i}^{\prime}$.

Let $f_{1}=1$ and let $\delta_{0}$ be any map. By induction assume that $f_{1}, \ldots, f_{i}$ and $\delta_{0}, \ldots, \delta_{i-1}$ have been chosen. If $S_{i}^{\prime} \neq \varnothing$, define $\delta_{i}: S_{i}^{\prime} \rightarrow \mathbf{R}^{+}$by $\delta_{i}(x)=d\left(f_{i}(x)\right.$, $\left.T_{i}^{\prime}\right)$ and, using Axiom 1, extend $\delta_{i}$ to a map: $U \rightarrow \mathbf{R}^{+}$. Choose $\delta_{i}$ arbitrarily if $S_{i}^{\prime}=\varnothing$. Then apply Corollary 2.10 to $S_{i+1}^{\prime}, T_{i+1}^{\prime}, \gamma_{i} / 2^{i}, U$, and $f_{i}$ to obtain $f_{i+1} \in V_{U}\left(f_{i}, \gamma_{i} / 2^{i}\right)$ such that $f_{i+1}\left(S_{i+1}^{\prime}\right) \cap T_{i+1}^{\prime}=\varnothing$. This completes the induction.

Lemma 2.11 will not be needed in its full strength until Theorem 2.15 , but the special case $S, T \in \delta_{+}$will be of use immediately.

Theorem 2.12. Let $\delta$ satisfy Axioms 1, 2, and 3 and let $B$ be a pseudo-boundary for $\mathcal{S}$ in $Y$. If $T \in \mathcal{S}_{+}$, then $B-T$ is a pseudo-boundary for $\mathcal{S}$ in $Y$. Thus if $U$ is an open subset of $Y$ and $\varepsilon: U \rightarrow \mathbf{R}^{+}$is a map, then there exists a homeomorphism $f \in V_{U}(1, \varepsilon)$ such that $f(B \cap U)=(B-T) \cap U$.

Proof. Clearly $B-T \in \mathcal{S}_{++}$. To prove that (*) holds, let $S \in \mathcal{S}$, let $U$ be open in $Y$, and let $\varepsilon: U \rightarrow \mathbf{R}^{+}$be a map. By Lemma 2.11 , there exists $f \in V_{U}(1, \varepsilon / 2)$ such that $f(S \cap U) \cap T=\varnothing$. Let $\delta: U \rightarrow \mathbf{R}^{+}$be a map such that $\delta(x)$ $=d(f(x), T)$ for each $x \in S \cap U$ and let $\gamma=\min \{\delta, \varepsilon / 2\}$. Since $B$ satisfies (**), there exists $h \in V_{U}(f, \gamma)$ such that $h(S \cap U) \subset B$. But then $h(S \cap U)$ $\subset B-T$ and $h \in V_{U}(1, \varepsilon)$. The second part of the theorem follows from 2.5.

Corollary 2.13. Let $\mathcal{S}$ satisfy Axioms 1,2 , and 3 and let $B$ be a pseudo-boundary for $\mathcal{S}$ in $Y$. If $T \in \mathcal{S}_{+}$, then $B \cap T$ is strongly negligible in $B$.

Proof. The proof is similar to that of 2.8 .

E. The maximal family generated by $\delta$. Suppose we start with an arbitrary subset $B$ of $Y$. Is $B$ a pseudo-boundary for some family $\delta$ ? The answer is, of course, yes. One need only use the absorption property $(*)$ to define the family $\delta$. However, the pseudo-boundary $B$ might not be very interesting since $\delta$ may not satisfy the axioms. We now consider what conditions on $B$ yield a family $\delta$ satisfying the axioms. Using 2.2 , the reader can easily prove 
Lemma 2.14. Let $B$ be a subset of $Y$ and let $\delta$ be the family of subsets $S$ of $Y$ such that whenever $U$ is open in $Y$ and $\varepsilon: U \rightarrow \mathbf{R}^{+}$is a map, there exists $h \in V_{U}(1, \varepsilon)$ such that $h(S \cap U) \subset B$.

(1) If for each $U$ open in $Y$, each $g \in H(Y)$, and each map $\varepsilon: g(U) \rightarrow \mathbf{R}^{+}$, there exists $h \in V_{g(U)}(1, \varepsilon)$ such that $g(B \cap U)=h(B \cap g(U))$, then 5 satisfies Axiom 2.

(2) If each subset of $B$ is thin in $Y$, then 5 satisfies Axiom 3.

Now let $\delta$ be a family of closed subsets of $Y$ and suppose we know that there exists a pseudo-boundary $B$ for $\delta$ in $Y$. We want to enlarge $\delta$ in order that the negligibility theorems can be applied to as large a family of sets as possible. Accordingly, let $\bar{\Im}$ denote the collection of all closed subsets $S$ of $Y$ such that for each open set $U$ in $Y$ and each map $\varepsilon: U \rightarrow \mathbf{R}^{+}$, there exists $h \in V_{U}(1, \varepsilon)$ such that $h(S \cap U) \subset B$. We call $\bar{\delta}$ the maximal family generated by $\delta$, because when $\delta$ satisfies Axioms 1 and $2, \bar{\delta}$ does not depend on the particular pseudo-boundary $B$ (by 2.5 ).

Theorem 2.15. Let $B$ be a pseudo-boundary for $\delta$ in $Y$. If $\delta$ satisfies Axioms 1 and 2 , then so does $\bar{\delta}$; and if $\mathcal{\delta}$ also satisfies Axiom 3, then so does $\bar{\delta}$. Moreover, $B$ is a pseudo-boundary for $\bar{\delta}$ in $Y$.

Proof. $\bar{\delta}$ satisfies Axiom 1 by definition. If $\delta$ satisfies Axioms 1 and 2, then 2.4 and 2.5 together imply that condition (1) of Lemma 2.14 holds. Hence $\bar{\delta}$ satisfies Axiom 2. If $\delta$ satisfies Axioms 1, 2, and 3, then 2.11 implies that condition (2) of Lemma 2.14 holds and hence that $\bar{\delta}$ satisfies Axiom 3.

Since $B$ is a pseudo-boundary for $\bar{\delta}$ in $Y$, the negligibility results 2.8 and 2.13 are true for the family $\overline{\mathcal{\delta}}$. In particular, we have

Corollary 2.16. Let $\delta$ satisfy Axioms 1,2 , and 3 and let $B$ be a pseudo-boundary for $\delta$ in $Y$. If $T$ is a closed subset of $Y$ which is contained in $B$, then $T$ is strongly negligible in $B$. Thus any compact subset of $B$ is strongly negligible in $B$.

Proof. $T \in \bar{\delta}$.

F. Additional limitations on $Y$. We examine here the pseudo-boundary structures when $Y$ satisfies additional topological properties, e.g., $\sigma$-compact or separable. This will greatly simplify our application of these results to euclidean spaces.

For a family $\delta$ of subsets of $Y$, let $\delta_{c}$ denote those elements of $\delta$ which are compact.

Lemma 2.17. Let $Y$ be $\sigma$-compact and let $\delta$ satisfy Axioms 1 and 2. If $B$ is $a$ pseudo-boundary for $\delta_{c}$ in $Y$, then $B$ is also a pseudo-boundary for $\delta$ in $Y$.

Proof. Clearly $\delta_{c}$ satisfies Axioms 1 and 2 and $B \in \delta_{++}$. If $S \in \delta$, then $S \in\left(\delta_{c}\right)_{++}$and hence 2.7 implies that absorption holds.

Lemma 2.18. Let $Y$ be $\sigma$-compact and let $\delta$ satisfy Axioms 1 and 2. If $\mathcal{S}_{c}$ satisfies Axiom 3, then so does 5 . 
Proof. Since $\delta_{c}$ satisfies Axioms 1, 2, and 3, Lemma 2.11 implies that any $S \in \mathcal{S} \subset\left(\delta_{c}\right)_{++}$is thin in $Y$.

Lemma 2.19. A compact subset $X$ of a metric space $Y$ is thin in $Y$ if and only if for each open set $U$ in $Y$ containing $X$ and each positive real number $\varepsilon$, there exists a homeomorphism $h \in H(Y)$ such that $h(X) \cap X=\varnothing, h$ fixes $Y-U$, and $d(x, h(x))<\varepsilon$ for each $x \in U$.

Proof. Elementary.

We now show that for separable metric spaces the absorption property takes on a particularly nice form. For this we will need the following lemma on starfinite open covers. It is proved, though not fully stated, as Theorem 2 of [3].

Lemma 2.20. Let $X$ be a topological space and $w$ a countable star-finite open cover of $X$. Then there exists an ordering $W_{1}, W_{2}, \ldots$ of the elements of $W$ and a sequence $\{n(i)\}_{i \geq 1}$ of positive integers with the following property: given any sequence $\left\{h_{i}\right\}_{i \geq 1}$ of homeomorphisms of $X$ such that $h_{i} \mid X-W_{i}=1$, there is a homeomorphism $h$ of $X$ such that $h$ agrees with $h_{k} \cdots h_{1}$ on $W_{i}$ for all $k \geq n(i)$.

Lemma 2.21. Let $Y$ be separable and let $\mathcal{S}$ satisfy Axiom 2. Then $B \in \mathcal{S}_{++}$is a pseudo-boundary for $\delta$ in $Y$ if and only if for each $S \in \mathcal{S}$, each open set $U$ in $Y$, and each $\varepsilon>0$, there exists a homeomorphism $h \in H(Y)$ such that $h$ fixes $Y-U$, $h(S \cap U) \subset B$, and $d(x, h(x))<\varepsilon$ for each $x \in U$.

Proof. Let $U$ be open in $Y$ and let $\varepsilon: U \rightarrow \mathbf{R}^{+}$be a map. For each $i \geq 1$, let $U_{i}=\varepsilon^{-1}((1 / i, \infty))$. Then $\left\{U_{i}\right\}_{i \geq 1}$ is an open cover of the separable metric space $U$ and hence has a countable star-finite refinement $\mho$. Let $W_{1}, W_{2}, \ldots$ be an ordering of the elements of $\mathscr{W}$ and let $\{n(i)\}_{i \geq 1}$ be a sequence of positive integers having the property stated in Lemma 2.20. For each $i \geq 1$, let $\varepsilon_{i}$ be a positive real number such that $\varepsilon_{i}<\inf \left\{\varepsilon(x) \mid x \in W_{i}\right\}$ and let

$$
\delta_{i}=\frac{\min \left\{\varepsilon_{1}, \ldots, \varepsilon_{i}\right\}}{2 \max \{n(1), \ldots, n(i)\}} .
$$

Using the hypotheses, it is easy to construct a sequence $\left\{h_{i}\right\}_{i \geq 1}$ of homeomorphisms of $Y$ such that

(1) $h_{i} \mid Y-W_{i}=1$,

(2) $d\left(x, h_{i}(x)\right)<\delta_{i}$ for each $x \in W_{i}$, and

(3) $h_{1}\left(S \cap W_{1}\right) \subset B$ and $h_{i+1}\left(h_{i}(S) \cap W_{i+1}\right) \subset B$ for each $i \geq 1$ (note that (3) uses Axiom 2). By Lemma 2.20, there exists $h \in H(Y)$ such that $h \mid W_{i}$ $=h_{n(i)} \cdots h_{1} \mid W_{i}$. Then $h \in V_{U}(1, \varepsilon)$ and $h(S \cap U) \subset B$. This proves the "if" part of the theorem. The "only if" part is obvious.

3. The universal pseudo-boundaries in $E^{n}$. For each integer $k,-1 \leq k<n$, we will define the universal $k$-dimensional pseudo-boundary of $E^{n}$. The appropriate family of sets, denoted abstractly by $\mathcal{S}$ in $\$ 2$, will be the family $\pi_{n}^{k}$ of strong $Z_{n-k-2^{-}}$-sets: they are defined and discussed in part A. Strong $Z_{n-k-2^{-}}$sets are the 
"tame" sets of dimension $\leq k$ in $E^{n}$ : every $k$-dimensional subpolyhedron of $E^{n}$ is a strong $Z_{n-k-2}$-set, and, if $k \leq n-3$ and $n \neq 4$, every $k$-dimensional compactum in $E^{n}$ whose complement is 1-ULC is a strong $Z_{n-k-2^{-}}$set. In part A we show that $\Re_{n}^{k}$ satisfies Axioms 1 and 2 of $\S 2$, and also Axiom 3 if $n \geq 2 k+1$. The verification of Axiom 2 depends primarily on engulfing theorems of Bryant [9] and [10], or alternatively of Štan'ko [29]. For certain values of $n$ and $k$ (usually low) we use Bing-Kister [6], McMillan [24], Gluck [17], and Bing [5].

In part B we define and establish the pseudo-boundaries. These are built out of universal $k$-dimensional compacta in $E^{n}([26]$, [30]). This motivates the name although we never use the fact, established only recently by Štan'ko [30], that they are universal. Our main source in part B is Bothe's paper [7]. For technical reasons we must develop his method from scratch, so the reader need not be familiar with [7].

In part $\mathbf{C}$ we apply $\$ 2$ to obtain many negligibility theorems. The section ends with formal proofs of Theorems 1.1 and 1.2.

A. Strong $Z_{n-k-2}$-sets. A closed subset $X$ of a topological space $Y$ is a $Z_{m}$-set ( $m$ an integer $\geq 0$ ) if for every nonempty $m$-connected open set $U$ in $Y, U-X$ is nonempty and $m$-connected (compare with the definition of $Z$-set [1]). If $Y$ is a metric space and $\varepsilon>0$, we denote the set of points whose distance from $X$ is less than $\varepsilon$ by $N(X, \varepsilon)$. An $\varepsilon$-push $h$ of the pair $(Y, X)$ is a homeomorphism $h$ of $Y$ for which an $\varepsilon$-isotopy $H$ of $Y$ exists satisfying: $H_{0}=1, H_{1}=h$, and $H_{t}$ $\mid Y-N(X, \varepsilon)=1$ for each $t \in[0,1]$. A closed subset $X$ of $E^{n}$ is a strong $Z_{m}$-set ( $m$ an integer, $-1 \leq m<n$ ) if for each compact subpolyhedron $P$ of $E^{n}$ having dimension $\leq m+1$, and each $\varepsilon>0$, there exists an $\varepsilon$-push $h$ of $\left(E^{n}, X \cap P\right)$ such that $h(X) \cap P=\varnothing$.

Throughout $\$ 3, k$ and $n$ will be integers, $n \geq 0,-1 \leq k<n$, and $\Re_{n}^{k}$ will be the family of all strong $Z_{n-k-2}$-sets in $E^{n}$. We will build a pseudo-boundary for $\pi_{n}^{k}$ in $E^{n}$. The family $\Re_{n}^{k}$ satisfies Axiom 1 of $\$ 2$ ("closed") by definition. Our first task (3.5) is to show that Axiom 2 of $\$ 2$ ("invariant") is also satisfied.

We first discussed the property strong $Z_{m}$ in our paper [15]. There we used a slightly different definition: specifically, $h$ was an $\varepsilon$-push of $\left(E^{n}, X\right)$ rather than of $\left(E^{n}, X \cap P\right)$. With this alteration we will call $X$ a strong $Z_{m}^{*}$-set. In fact, the two definitions are equivalent (see 3.4), but the new one is more convenient to work with.

Proposition 3.1. Let $X$ be a closed subset of $E^{n}$.

(1) If $X$ is a strong $Z_{n-k-2}$-set, then $\operatorname{dim} X \leq k$;

(2) if $k=n-1$ and $\operatorname{dim} X \leq k$, then $X$ is a strong $Z_{n-k-2}$-set;

(3) if $k=n-2, n \neq 3$, and $\operatorname{dim} X \leq k$, then $X$ is a strong $Z_{n-k-2}$-set;

(4) if $k \leq n-3, n \neq 4, \operatorname{dim} X \leq k$, and $X$ is a $Z_{1^{-}}$-set, then $X$ is a strong $Z_{n-k-2}$ set;

(5) if $(n, k) \neq(3,1),(4,0)$ or $(4,1)$, and $X$ is a $Z_{n-k-2}$-set, then $X$ is a strong $Z_{n-k-2}-$ set. 
The proof is by means of three lemmas.

Lemma 3.2. If in Proposition 3.1, the term "strong $Z_{n-k-2}$-set" is everywhere replaced by "strong $Z_{n-k-2}^{*}$-set", then a true proposition results.

Proof. If $k=-1$, then $X=\varnothing$ and the lemma is trivial. Assume $k \geq 0$. For (1), take arbitrarily small polyhedral neighborhoods of $X$, push $X$ off the dual $(n-k-1)$-skeleton and then towards the $k$-skeleton: this implies $\operatorname{dim} X \leq k$. (2) is obvious. (3) is an easy application of the Bing-Kister homeomorphism extension theorem for (1-dimensional) polyhedra in the trivial range [6] when $n \geq 4$; (3) follows from the classical Schoenflies theorem when $n=2$. (4) follows from theorems of Bryant [9], [10], Stan'ko [29], and McMillan [24]: precisely how it follows is set out in the proof of Theorem 2.2 of [15]. In connection with (4), note that when $X$ is compact and $\operatorname{dim} X<n$, then $X$ is a $Z_{1}$-set if and only if $E^{n}-X$ is uniformly locally simply connected (1-ULC). (5) reduces to (4) if $k \leq n-3$ (see Theorem 2.3 of [15]); (5) reduces to (3) if $k=n-2$; (5) is obvious if $k=n-1$.

Lemma 3.3. If $X$ is a strong $Z_{n-k-2}^{*}$-set and $h$ is a homeomorphism of $E^{n}$, then $h(X)$ is also a strong $Z_{n-k-2}^{*}-$ set.

Proof. This follows from 3.2 except for three special cases: if $(n, k)=(4,0)$ or $(4,1)$, it follows from Theorem 1.1 of [17]; if $(n, k)=(3,1)$, it follows immediately from the three-dimensional "hauptvermutung", see for example Theorem 2 of [5].

Lemma 3.4. Let $X$ be a subset of $E^{n}$. Then $X$ is a strong $Z_{n-k-2}$-set if and only if it is a strong $Z_{n-k-2}^{*}$-set.

Proof. On pp. 251-252 of [29], Štan'ko gives a simple geometrical proof which depends only on Lemma 3.3 above. When $X$ is compact, Stan'ko's terminology is related to ours as follows: $X$ is a strong $Z_{n-k-2}$-set if and only if Dem $X \leq k$; $X$ is a strong $Z_{n-k-2}^{*}$-set if and only if $\operatorname{dem} X \leq k$.

Proof of Proposition 3.1. Combine 3.2 and 3.4.

Note. Proposition 3.1(3) is false when $n=3$. Counterexamples have been given by Bothe [8] and McMillan-Row [25].

Corollary 3.5. The family $\Re_{n}^{k}$ satisfies Axiom 2.

Proof. Combine 3.3 and 3.4.

Our next goal is to show that $\mathfrak{T}_{n}^{k}$ satisfies Axiom 3 whenever $n \geq 2 k+1$ (Lemma 3.8).

Lemma 3.6. The finite union of strong $Z_{n-k-2}$-sets in $E^{n}$ is a strong $Z_{n-k-2}$-set.

Proof. We need only consider the union of two strong $Z_{n-k-2}$-sets. Let $X$ and $Y$ be strong $Z_{n-k-2^{-}}$sets in $E^{n}$, let $U$ be an open subset of $E^{n}$ containing $(X \cup Y) \cap P$, where $P$ is a compact subpolyhedron of $E^{n}$ having dimension $\leq n-k-1$, and let $\varepsilon>0$. Let $G_{t}$ be an $\varepsilon / 2$-isotopy of $E^{n}$, fixed outside $U$, such 
that $G_{0}=1$ and $G_{1}(X) \cap P=\varnothing$. Let $0<\delta<\min \left\{\varepsilon / 2, d\left(G_{1}(X), P\right)\right\}$. By 3.5, $G_{1}(Y)$ is a strong $Z_{n-k-2^{-}}$set in $E^{n}$ and $G_{1}(Y) \cap P \subset U$. Hence there is a $\delta$ isotopy $F_{t}$ of $E^{n}$, fixed outside $U$, such that $F_{0}=1$ and $F_{1}\left(G_{1}(Y) \cap P\right)=\varnothing$. Set $H_{t}=F_{t} G_{t}$ and note that $H_{1}(X \cup Y) \cap P=\varnothing$.

Lemma 3.7. $A$ closed subset of a strong $Z_{n-k-2}$-set in $E^{n}$ is a strong $Z_{n-k-2}$-set.

Proof. Let $X^{\prime}$ be a closed subset of a strong $Z_{n-k-2}$-set $X$ and let $P$ be a compact subpolyhedron of $E^{n}$ having dimension $\leq n-k-1$. If $U$ is an open subset of $E^{n}$ containing $P \cap X^{\prime}$, there is a compact subpolyhedron $P^{\prime}$ of $P$ such that $P \cap X^{\prime} \subset P^{\prime} \subset U$. Then an isotopy which pushes $X$ off $P^{\prime}$ while fixing $E^{n}-U$ and moving points a small distance will push $P$ off $X^{\prime}$ in the desired fashion.

Lemma 3.8. If $n \geq 2 k+1$, then $\mathfrak{\pi}_{n}^{k}$ satisfies Axiom 3 .

Proof. If $k=-1$, then $\Re_{n}^{k}=\{\varnothing\}$ and the lemma is trivial. Assume $k \geq 0$. By 3.6 and 3.7, we need only prove that a strong $Z_{n-k-2}$-set $X$ is thin in $E^{n}$. Moreover, by 2.18 , we may assume that $X$ is compact. Let $U$ be open in $E^{n}$ containing $X$ and let $\varepsilon>0$. We may assume that $\varepsilon / 4<d\left(X, E^{n}-U\right)$. Let $N$ be a compact polyhedral neighborhood of $X$ contained in $U$ and let $T$ be a triangulation of $N$ such that mesh $T<\varepsilon / 4$. Let $T^{k}$ denote the $k$-skeleton of $T$ and $\tilde{T}^{n-k-1}$ the dual $(n-k-1)$-skeleton. Since $\left|T^{k}\right|$ and $\left|\tilde{T}^{n-k-1}\right|$ are nonempty and disjoint, there exist disjoint open sets $V$ and $W$ containing $\left|T^{k}\right|$ and $\left|\tilde{T}^{n-k-1}\right|$, respectively. Let $\gamma<\min \left\{\varepsilon / 4, d\left(X, E^{n}-\operatorname{Int} N\right)\right\}$. Since $X$ is a strong $Z_{n-k-2^{-s e t}}$ in $E^{n}$ there is a $\gamma$-push $h_{1}$ of $\left(E^{n}, X \cap\left|\tilde{T}^{n-k-1}\right|\right)$ such that $h_{1}(X) \cap\left|\tilde{T}^{n-k-1}\right|=\varnothing$. But $\left|T^{k}\right|$ and $\left|\tilde{T}^{n-k-1}\right|$ are complementary skeleta and hence there is an $\varepsilon / 4$-push $h_{2}$ of $\left(E^{n}, h_{1}(X)\right)$ such that $h_{2} h_{1}(X) \subset V$. Similarly, since $k \leq n-k-1$, there is a $\gamma$-push $h_{3}$ of $\left(E^{n}, X \cap\left|T^{k}\right|\right)$ such that $h_{3}(X) \cap\left|T^{k}\right|=\varnothing$ and an $\varepsilon / 4$-push $h_{4}$ of $\left(E^{n}, h_{3}(X)\right)$ such that $h_{4} h_{3}(X) \subset W$. Then $h_{4} h_{3}(X) \cap h_{2} h_{1}(X)=\varnothing$ and hence $h=h_{3}^{-1} h_{4}^{-1} h_{2} h_{1}$ is a homeomorphism of $E^{n}$ fixing $E^{n}-U$ such that $h(X) \cap X=\varnothing$ and $d(x, h(x))<\varepsilon$ for each $x \in U$. By Lemma 2.19, $X$ is thin in $E^{n}$.

Lemma 3.9. If $X$ is a closed subset of $E^{n}$ which is the countable union of strong $Z_{n-k-2}$-sets, then $X$ is a strong $Z_{n-k-2}$-set in $E^{n}$.

Proof. Write $X=\cup_{i=1}^{\infty} X_{i}$ where each $X_{i}$ is a strong $Z_{n-k-2}$-set and let $P$ be a compact $(n-k-1)$-dimensional subpolyhedron of $E^{n}$. Push $X_{1}$ off $P$, then (using 3.5) push $X_{2}$ off $P$ keeping $X_{1}$ away from $P$, and so on. If the pushes are chosen small enough, their limit will be a homeomorphism as required. The details are similar to those in the proof of Lemma 2.11.

Our next lemma will be needed in part B. Let $N^{n-k-1}$ be the set of all points $\left(x_{1}, \ldots, x_{n}\right)$ in $E^{n}$ for which at least $k+1$ of the coordinates $x_{r}$ are rational. Then $N^{n-k-1}$ is the countable union of $(n-k-1)$-dimensional planes in $E^{n}$.

Lemma 3.10. Let $X$ be a strong $Z_{n-k-2}$-set in $E^{n}$, let $U$ be an open subset of $E^{n}$, 
and let $\varepsilon>0$. Then there is a homeomorphism $h$ of $E^{n}$ fixing $E^{n}-U$ such that $h(X) \cap U \cap N^{n-k-1}=\varnothing$ and $d(x, h(x))<\varepsilon$ for each $x \in X$.

Proof. Write $N^{n-k-1} \cap U$ as the countable union of compact $(n-k-1)$ dimensional subpolyhedra $P_{i}$ of $E^{n}$ and push $X$ off each successive $P_{i}$. Again, the details are similar to those in the proof of 2.11 .

B. Construction of the pseudo-boundary. It will be convenient in part B to use a cell complex structure on euclidean space, instead of the usual PL triangulations. We will fix this structure throughout part B and define all homeomorphisms in terms of the cells. For any integer $i \geq 1$, let $J_{i}$ denote the (infinite) cell complex in $E^{n}$ whose $n$-cells have the form

$$
\left[\frac{l_{1}}{3^{i-1}}, \frac{l_{1}+1}{3^{i-1}}\right] \times\left[\frac{l_{2}}{3^{i-1}}, \frac{l_{2}+1}{3^{i-1}}\right] \times \cdots \times\left[\frac{l_{n}}{3^{i-1}}, \frac{l_{n}+1}{3^{i-1}}\right]
$$

where $l_{1}, \ldots, l_{n}$ are integers, and whose faces are described in the obvious fashion. By a complex $K$ in $J_{i}$ we mean a collection of cells of $J_{i}$ such that if $\sigma \in K$ and $\tau$ is a face of $\sigma$, then $\tau \in K$. A subcomplex $L$ of a complex $K$ in $J_{i}$ is a subset of $K$ which is a complex in $J_{i}$. If $L$ is a subcomplex of $K$ in $J_{i}$, we let $\Re(L, K)$ be the collection of all $\sigma \in K$ such that there exist $\tau \in K$ and $\gamma \in L$ with $\sigma$ and $\gamma$ faces of $\tau$, i.e., $\vartheta(L, K)$ consist of all cells in $K$ which meet $L$, together with their faces. $|K|$ denotes the union of all cells in $K$.

If $K$ is a complex in $J_{i}$, let sd $K$ denote the collection of all cells in $J_{i+1}$ which lie in a cell of $K$. Clearly sd $K$ is a complex in $J_{i+1}$ and $|\operatorname{sd} K|=|K|$.

Lomma 3.11. If $K$ is a complex in $J_{i}$, then $\mid \Re\left(\right.$ sd $\left.K, J_{i+1}\right) \mid$ is a regular neighborhood of $|K|$ in the usual $P L$ sense and hence is an n-manifold with boundary in $E^{n}$.

Proof. Note that the cells of $J_{i+1}$ are obtained from those of $J_{i}$ by dividing all edges into thirds. The lemma can therefore be proved by combining three results from [20], namely $1.4,2.6$ and $2.11(1)$.

If $K$ is a cell complex in $J_{i}$, let $K^{k}$ denote the $k$-skeleton of $K$, i.e., the collection of all cells having dimension $\leq k$.

For each integer $j \geq 1$, let $K_{j}=\Re\left(\operatorname{sd} J_{j}^{k}, J_{j+1}\right)$ and then set $B_{i}\left(\Re_{n}^{k}\right)$ $=\bigcap_{j=i}^{\infty}\left|K_{j}\right|$ for each integer $i \geq 1$. Since $B_{i}\left(\Re_{n}^{k}\right)=\left|K_{i}\right| \cap B_{i+1}\left(\Re_{n}^{k}\right)$, we have $B_{i}\left(\Re_{n}^{k}\right) \subset B_{i+1}\left(\Re_{n}^{k}\right)$. We call $B\left(\Re_{n}^{k}\right)=\cup_{i=1}^{\infty} B_{i}\left(\Re_{n}^{k}\right)$ the universal $k$-dimensional pseudo-boundary in $E^{n}$.

Theorem 3.12. $B\left(\Re_{n}^{k}\right)$ is a pseudo-boundary for $\Re_{n}^{k}$ in $E^{n}$.

The proof of this theorem will take up the remainder of part B. Since each $\left|K_{j}\right|$ is a regular neighborhood of a $k$-dimensional polyhedron, a simple general position argument implies that $B_{i}\left(\Re_{n}^{k}\right)$ is a strong $Z_{n-k-2}$-set in $E^{n}$. So it remains to show that $B\left(\mathscr{N}_{n}^{k}\right)$ has the absorption property. Lemmas 2.17 and 2.21 reduce the problem to the following:

Theorem 3.12'. Let $X$ be a compact strong $Z_{n-k-2}$-set in $E^{n}$, let $U$ be an open 
subset of $E^{n}$ with compact closure, and let $\varepsilon>0$. Then there is a homeomorphism $h: E^{n} \rightarrow E^{n}$ fixing $E^{n}-U$ such that $h(X \cap U) \subset B\left(\Re_{n}^{k}\right)$ and $d(x, h(x))<\varepsilon$ for each $x \in U$.

We have already mentioned that the space $B_{1}\left(\Re_{n}^{k}\right)$ was first defined by Menger in [26]. He asserted, and Lefschetz subsequently proved in [23], that when $n=2 k+1$, any compactum of dimension $\leq k$ can be embedded in $B_{1}\left(\Re_{n}^{k}\right)$. In [7], Bothe improved this greatly. Letting $N^{n-k-1}$ be as in our Lemma 3.10, and simply assuming $k<n$, Bothe proved that if $X$ is a compact subset of $E^{n}-N^{n-k-1}$, then there is a homeomorphism $h$ of $E^{n}$ such that $h(X) \subset B_{1}\left(\Re_{n}^{k}\right)$. Together with Lemma 3.10, this implies that compact strong $Z_{n-k-2}$-sets in $E^{n}$ can be moved into $B_{1}\left(\mathscr{T}_{n}^{k}\right)$ by ambient homeomorphisms. The relevance of this to Theorem 3.12' is obvious. The basic idea of using the homeomorphism $\varphi_{j}^{e}$ (defined below) on each real factor of $E^{n}$ is of course Bothe's. Our aim in what follows is to set up enough of his apparatus to prove Theorem 3.12'.

We remark in passing that Stan'ko in [30] has proved that if $X$ is a compact subset of $E^{n}$ and $\operatorname{dim} X \leq n-3$, then there is another copy $X^{\prime}$ of $X$ in $E^{n}$ such that $E^{n}-X^{\prime}$ is 1-ULC. This fact, together with Proposition 3.1 and the above remarks, implies that every $k$-dimensional compact subset of $E^{n}$ can be embedded in $B_{1}\left(\Re_{n}^{k}\right)$. This in brief is Štan'ko's solution to a famous problem posed by Menger (see [21, Remark, p. 65]).

Let $E^{n}$ carry the "maximum metric" $d(x, y)=\max \left\{\mid x_{i}-y_{i} \| 1 \leq i \leq n\right\}$. Let

$$
N^{n-k-1}=\left\{x \in E^{n} \mid \text { at least } k+1 \text { of the coordinates } x_{r} \text { of } x \text { are rational }\right\} .
$$

Let

$L_{j}^{n-k-1}=\left\{x \in E^{n} \mid\right.$ at least $k+1$ of the coordinates $x_{r}$ have the form

$$
\left.\left(l_{r}+\frac{1}{2}\right) / 3^{j-1} \text { for some integer } l_{r}\right\} \text {. }
$$

Then $L_{j}^{n-k-1} \subset N^{n-k-1}$ for each $j \geq 1$. Note that

$\left|J_{j}^{k}\right|=\left\{x \in E^{n} \mid\right.$ at least $n-k$ of the coordinates $x_{r}$ have the form

$$
\left.l_{r} / 3^{j-1} \text { for some integer } l_{r}\right\} \text {. }
$$

We now state and prove several technical lemmas which will be useful later.

Lemma 3.13. $A$ point $x$ lies in $\left|K_{j}\right|$ if and only if for at least $(n-k)$ coordinates $x_{r}, l_{r} / 3^{j} \leq x_{r} \leq\left(l_{r}+1\right) / 3^{j}$ where $l_{r}$ is an integer and 3 divides either $l_{r}$ or $l_{r}+1$.

Proof. $\left|K_{j}\right|$ is the union of all $n$-cells of $J_{j+1}$ which meet a $k$-dimensional plane of $\left|J_{j}^{k}\right|$. A $k$-plane of $J_{j}$ has the form $\left\{x \in E^{n} \mid\right.$ for a subset $B$ of $\{1, \ldots, n\}$ consisting of $n-k$ integers, $x_{r}=l_{r}^{\prime} / 3^{j-1}$ for each $r \in B$ and $x_{r}$ is arbitrary for $r \notin B\}$ and an $n$-cell of $J_{i+1}$ has the form $\prod_{r=1}^{n}\left[l_{r} / 3^{j-1},\left(l_{r}+1\right) / 3^{j-1}\right]$. These can intersect if and only if either $l_{r}$ or $l_{r}+1$ is equal to $3 l_{r}^{\prime}$ for each $r \in B$. 
Lemma 3.14. Let $\varepsilon>0$, let $j \geq 1$ be an integer, and let $x \in E^{n}$. Then $d\left(x, L_{j}^{n-k-1}\right) \geq \varepsilon / 3^{j-1}$ if and only if for at least $n-k$ coordinates $x_{r} \mid x_{r}$ $-\left(l_{r}+\frac{1}{2}\right) / 3^{j-1} \mid \geq \varepsilon / 3^{j-1}$ for some integer $l_{r}$.

Proof. By contradiction.

For each $\varepsilon \in(0,1 / 6]$ and each integer $j \geq 1$, define $\varphi_{j}^{e}: \mathbf{R} \rightarrow \mathbf{R}$ to be the PL homeomorphism such that for all integers $l$,

(1) $\varphi_{j}^{e}$ fixes $l / 3^{j-1}$ and $\left(l+\frac{1}{2}\right) / 3^{j-1}$;

(2) $\varphi_{j}^{\varepsilon}\left(\left(l+\frac{1}{2}-\varepsilon\right) / 3^{j-1}\right)=\left(l+\frac{1}{3}\right) / 3^{j-1}$;

(3) $\varphi_{j}^{e}\left(\left(l+\frac{1}{2}+\varepsilon\right) / 3^{j-1}\right)=\left(l+\frac{2}{3}\right) / 3^{j-1}$;

(4) $\varphi_{j}^{\varepsilon}$ is linear on the intervals formed by the partition $\left\{l / 3^{j-1},\left(l+\frac{1}{2} \pm \varepsilon\right) / 3^{j-1}\right\}$ of $\mathbf{R}$.

If $\varepsilon$ is a rational number, then $\varphi_{j}^{e}(\mathbf{Q})=\mathbf{Q}$, where $\mathbf{Q}$ denotes the set of rational numbers. Now let $i \geq 1$ be an integer and let $\gamma=\left\{\varepsilon_{j}\right\}_{j \geq i}$ be a sequence of real numbers in $(0,1 / 6]$. Define $\psi_{j}^{i, \gamma}: \mathbf{R} \rightarrow \mathbf{R}$ by $\psi_{j}^{i, \gamma}=\varphi_{j}^{\varepsilon_{j}} \cdots \varphi_{i}^{e_{i}}$ for each $j \geq i$.

Lemma 3.15. For any integer $i \geq 1, \psi^{i, \gamma}=\lim _{j \rightarrow \infty, j \geq i} \psi_{j}^{i, \gamma}$ is a homeomorphism of $\mathbf{R}$, and for each integer $l$, $\psi^{i, \gamma}$ leaves the interval $\left[l / 3^{i-1},(l+1) / 3^{i-1}\right]$ invariant.

Proof. Routine. See p. 212 of [7].

Identifying $E^{n}$ with the $n$-fold product $\mathbf{R}^{n}$ we have the following homeomorphisms of $E^{n}: \Phi_{j}^{e}=\varphi_{j}^{e} \times \cdots \times \varphi_{j}^{e}, \Psi_{j}^{i, \gamma}=\psi_{j}^{i, \gamma} \times \cdots \times \psi_{j}^{i, \gamma}$, and $\Psi^{i, \gamma}=\psi^{i, \gamma} \times \cdots$ $\times \psi^{i, \gamma}$. Observe that $\Psi^{i, \gamma}=\lim _{j \rightarrow \infty, j \geq i} \Psi_{j}^{i, \gamma}$. Note that $\Psi^{i, \gamma}$ leaves each cell in $J_{j}$ invariant when $j \leq i$; in particular $\Psi^{i, \gamma}\left(\left|K_{j}\right|\right)=\left|K_{j}\right|$ when $j<i$. Note also $d\left(x, \Psi^{i, \gamma}(x)\right)<1 / 3^{i-1}$.

Lemma 3.16. Let $\varepsilon \in(0,1 / 6]$ and let $C$ denote the set of points $x$ in $E^{n}$ such that $d\left(x, L_{j}^{n-k-1}\right) \geq \varepsilon / 3^{j-1}$. Then $\Phi_{j}^{\varepsilon}(C) \subset\left|K_{j}\right|$.

Proof. Let $x \in C$. Then $\Phi_{j}^{\varepsilon}(x)=\left(\varphi_{j}^{e}\left(x_{1}\right), \ldots, \varphi_{j}^{e}\left(x_{n}\right)\right)$. By Lemma 3.14 there exists $A \subset\{1, \ldots, n\}$ containing $n-k$ integers such that $\mid x_{r}-\left(l_{r}+\frac{1}{2}\right) / 3^{j-4}$ $\geq \varepsilon / 3^{j-1}$ for each $r \in A$. If $x_{r} \leq\left(l_{r}+\frac{1}{2}-\varepsilon\right) / 3^{j-1}$, then

$$
\varphi_{j}^{\epsilon}\left(x_{r}\right) \in\left[3 l_{r} / 3^{j},\left(3 l_{r}+1\right) / 3^{j}\right]
$$

and if $x_{r} \geq\left(l_{r}+\frac{1}{2}+\varepsilon\right) / 3^{j-1}$, then

$$
\varphi_{j}^{\epsilon}\left(x_{r}\right) \in\left[\left(3 l_{r}+2\right) / 3^{i}, 3\left(l_{r}+1\right) / 3^{j}\right] .
$$

Thus Lemma 3.13 implies that $\Phi_{j}^{\varepsilon}(C) \subset\left|K_{j}\right|$.

Proof of Theorem 3.12'. By Lemma 3.10 we may assume $X \cap N^{n-k-1} \cap U$ $=\varnothing$. Using Lemma 3.11, there is an increasing sequence of positive integers $i_{1}, i_{2}, \ldots$ and a sequence of complexes $M_{i_{r}}$ in $J_{i_{r}}$ such that

(1) mesh $J_{i_{1}}=1 / 3^{i_{1}-1}<\varepsilon / 6$,

(2) each $\left|M_{i}\right|$ is a compact $n$-manifold with boundary in $E^{n}$,

(3) $\left|M_{i_{r}}\right| \subset$ Int $\left|M_{i_{r+1}}\right|$ for each $r \geq 1$, and

(4) $U=\cup_{r=1}^{\infty}\left|M_{i_{r}}\right|$. 
Let $X_{i_{r}}=X \cap\left|M_{i_{r}}\right|$ and let $h_{1}=1 . X_{i_{1}}$ is compact and misses $L_{i_{1}}^{n-k-1}$, so there is a rational number $\epsilon\left(i_{1}\right) \in(0,1 / 6]$ such that $d\left(X_{i_{1}}, L_{i_{1}}^{n-k-1}\right) \geqslant$ $\epsilon\left(i_{1}\right) / 3^{i_{1}-1}$. Then $\Phi_{i_{1}}^{\epsilon\left(i_{1}\right)}$ is a homeomorphism of $E^{n}$ and $\Phi_{i_{1}}^{\epsilon\left(i_{1}\right)}\left(X_{i_{1}}\right) \subset\left|K_{i_{1}}\right|$ by Lemma 3.16. Since $\epsilon\left(i_{1}\right)$ is rational $\Phi_{i_{1}}^{\epsilon\left(i_{1}\right)}\left(N^{n-k-1}\right)=N^{n-k-1}$, and hence there is a rational number $\epsilon\left(i_{1}+1\right)$ such that $d\left(\Phi_{i_{1}}^{\epsilon\left(i_{1}\right)}\left(X_{i_{1}}\right), L_{i_{1}+1}^{n-k-1}\right)$ $\geq \varepsilon\left(i_{1}+1\right) / 3^{i_{1}}$. Lemma 3.16 and the fact that $K_{i_{1}}$ is a complex in $J_{i_{1}+1}$ imply $\Phi_{i_{1}+1}{ }^{e\left(i_{1}+1\right)} \Phi_{i_{1}}^{e\left(i_{1}\right)}\left(X_{i_{1}}\right) \subset\left|K_{i_{1}}\right| \cap\left|K_{i_{1}+1}\right|$. By induction choose rationals $\varepsilon\left(i_{1}\right), \varepsilon\left(i_{1}\right.$ $+1), \ldots, \varepsilon\left(i_{2}-1\right)$ such that $\Phi_{i_{2}-1}{ }^{e\left(i_{2}-1\right)} \ldots \Phi_{i_{1}}^{e\left(i_{1}\right)}\left(X_{i_{1}}\right) \subset \bigcap_{j=i_{1}}^{i_{2}-1}\left|K_{j}\right|$. All these homeomorphisms leave $\left|M_{i_{1}}\right|$ invariant. Define a homeomorphism $h_{2}$ of $E^{n}$ which agrees with $\Phi_{i_{2}-1}{ }^{e\left(i_{2}-1\right)} \cdots \Phi_{i_{1}}^{e\left(i_{1}\right)}$ on $\left|M_{i_{1}}\right|$, is the identity on $E^{n}-$ Int $\left|M_{i_{2}}\right|$, and satisfies $d\left(x, h_{2}(x)\right)<\varepsilon / 2$ for each $x \in E^{n}$; this can be done because $h_{2}|\mathrm{Bd}| M_{i_{1}} \mid$ is ambient isotopic to $1_{\mathrm{Bd}\left|M_{i}\right|}$ and a small compact boundary collar outside $\left|M_{i_{1}}\right|$ can be used to achieve the desired extension. By $3.5, h_{2}(X)$ is a strong $Z_{n-k-2^{2}}$-set in $E^{n}$. By applying 3.10 to the open set Int $\left|M_{i_{2}}\right|-\left|M_{i_{1}}\right|$, we may assume that $h_{2}(X) \cap N^{n-k-1} \cap U=\varnothing$.

Now we continue this procedure using $h_{2}\left(X_{i_{2}}\right)=h_{2}(X) \cap\left|M_{i_{2}}\right|$. Let $\epsilon\left(i_{2}\right)$ $\in(0,1 / 6]$ be a rational number such that $d\left(h_{2}\left(X_{i_{2}}\right), L_{i_{2}}^{n-k-1}\right) \geq \varepsilon\left(i_{2}\right)$. Then $\Phi_{i_{2}}^{e\left(i_{2}\right)}\left(h_{2}\left(X_{i_{2}}\right)\right) \subset\left|K_{i_{2}}\right|$ and $\Phi_{i_{2}}^{e\left(i_{2}\right)}\left(h_{2}\left(X_{i_{1}}\right)\right) \subset \bigcap_{j=i_{1}}^{i_{2}}\left|K_{j}\right|$. By induction choose rationals $\varepsilon\left(i_{2}\right), \ldots, \varepsilon\left(i_{3}-1\right)$ in $(0,1 / 6]$ and a homeomorphism $h_{3}$ of $E^{n}$ which agrees with $\Phi_{i_{3}-1}{ }^{e\left(i_{3}-1\right)} \cdots \Phi_{i_{2}}^{e\left(i_{2}\right)} h_{2}$ on $\left|M_{i_{2}}\right|$, is the identity on $E^{n}$ - Int $\left|M_{i_{3}}\right|$, and satisfies $d\left(x, h_{3}(x)\right)<\varepsilon / 2$ for each $x \in E^{n}$. Again assume $h_{3}(X) \cap N^{n-k-1} \cap U=\varnothing$. We have $h_{3}\left(X_{i_{2}}\right) \subset \bigcap_{j=i_{2}}^{i_{3}-1}\left|K_{j}\right|$ and $h_{3}\left(X_{i_{1}}\right) \subset \bigcap_{j=i_{1}}^{i_{3}-1}\left|K_{j}\right|$.

Repetition will yield rationals $\gamma=\{\epsilon(j)\}_{j \geqslant i_{1}} \subset(0,1 / 6]$ and homeomorphisms $\left\{h_{r}\right\}_{r \geq 2}$ such that $h_{r}$ agrees with $\Phi_{i_{r}-1}^{\left(i_{r}-1\right)} \cdots \Phi_{i_{r-1}}^{e\left(i_{r-1}\right)} h_{r-1}$ on $\left|M_{i_{r-1}}\right|, h_{r}$ fixes $E^{n}$ - Int $\left|M_{i_{r}}\right|, d\left(x, h_{r}(x)\right)<\varepsilon / 2$ for each $x \in E^{n}$, and $h_{s}\left(X_{i_{r}}\right) \subset \bigcap_{j=i_{r}}^{i_{s}-1}\left|K_{j}\right|$ where $1 \leq r<s$. The limit homeomorphism $h=\lim _{i \rightarrow \infty} h_{i} \cdots h_{1}$ exists: in fact, $h$ agrees with $\Psi^{i_{r}, \gamma} h_{r}$ on $\left|M_{i_{r}}\right|$ (which implies $d(x, h(x))<\epsilon$ if $x \in U$ ) and $h$ fixes $E^{n}-U$. Since $X_{i_{r}} \subset\left|M_{i_{s}}\right|$ when $s>r$ and $\Psi^{i_{s}, \gamma}\left(\left|K_{j}\right|\right)=\left|K_{j}\right|$ when $i_{s}>j$ we have $h\left(X_{i_{r}}\right)=\Psi^{i_{s}, \gamma} h_{s}\left(X_{i_{r}}\right) \subset \bigcap_{j=i_{r}}^{i_{s}-1}\left|K_{j}\right|$; thus $h\left(X_{i_{r}}\right) \subset B_{i_{r}}\left(\Re_{n}^{k}\right)$ implying $h(X$ $\cap U) \subset B\left(\Re_{n}^{k}\right)$.

Note. Lemma 3.9 implies that the maximal family $\overline{\mathscr{N}}_{n}^{k}$ generated by $\mathfrak{N}_{n}^{k}$ is simply $\mathfrak{\pi}_{n^{*}}^{k}$

C. Negligibility results. Now we apply $\$ 2$ to the present situation.

Let $P\left(\Re_{n}^{k}\right)=E^{n}-B\left(\Re_{n}^{k}\right) .2 .8,3.5$ and 3.12 yield

Theorem 3.17. Let $X$ be the countable union of strong $Z_{n-k-2}$-sets in $E^{n}$. Then $P\left(\Re_{n}^{k}\right) \cap X$ is strongly negligible in $P\left(\Re_{n}^{k}\right)$.

Theorem 3.18. Let $X$ be a subset of $P\left(\Re_{n}^{k}\right)$ and suppose that either

(1) $n \leq 2 k+1$, or

(2) $k \geq 2$ and $\operatorname{dim} X \leq k$.

If $X$ is an $F_{\sigma}$ in $E^{n}$, then $X$ is strongly negligible in $P\left(\Re_{n}^{k}\right)$. In particular, if $X$ is a o-compact subset of $P\left(\Re_{n}^{k}\right)$, then $X$ is strongly negligible in $P\left(\Re_{n}^{k}\right)$.

Proof. Suppose $X^{\prime}$ is a closed subset of $E^{n}$ lying in $P\left(\Re_{n}^{k}\right)$. If $n \leq 2 k+1$, then 
any compact subpolyhedron of $E^{n}$ having dimension $\leq n-k-1 \leq k$ can be absorbed into $B\left(\Re_{n}^{k}\right)$ and hence can be pushed off $X^{\prime}$. If $k \geq 2$ and $\operatorname{dim} X^{\prime} \leq k$, then Proposition 3.1 can be used (absorb a singular 2-disk into $B\left(\Re_{n}^{k}\right)$ in the case $k \leq n-3)$ to show that $X^{\prime}$ is a strong $Z_{n-k-2^{-}}$-set. In either case, $X^{\prime} \in \pi_{n}^{k}$ and the theorem follows from 3.17.

A simple special argument also gives Theorem 3.18 for the case $n=2, k=0$.

Lemma 3.19. Let $U$ be an open subset of $E^{n}$. Then $U$ is nonempty and $(n-k-2)$ connected if and only if $U \cap P\left(\pi_{n}^{k}\right)$ is nonempty and $(n-k-2)$-connected.

Proof. If $U$ is $(n-k-2)$-connected and $\partial \gamma$ is a singular $(n-k-2)$-sphere in $U \cap P\left(\Re_{k}^{n}\right)$, let $\partial \gamma$ bound a singular $(n-k-1)$-ball $\gamma$ in $U$. By Lemma 4.2 of [20], we may assume that $\gamma-\partial \gamma$ is "polyhedral" and hence may write $\gamma-\partial \gamma$ as the countable union of compact $(n-k-1)$-dimensional subpolyhedra of $E^{n}$. But $B\left(\Re_{n}^{k}\right)$ is the countable union of strong $Z_{n-k-2}$-sets in $E^{n}$ and therefore $\gamma$ can be pushed off $B\left(\Re_{n}^{k}\right)$ fixing $\partial \gamma$ and $E^{n}-U$ (see the proofs of 2.11, 3.9, and 3.10). This proves that $U \cap P\left(\Re_{n}^{k}\right)$ is $(n-k-2)$-connected.

If $U \cap P\left(\Re_{n}^{k}\right)$ is $(n-k-2)$-connected and $\partial \gamma$ is a PL singular $(n-k-2)$ sphere in $U$, then $\partial \gamma$ can be pushed off $B\left(\Re_{n}^{k}\right)$ into $U \cap P\left(\Re_{n}^{k}\right)$. In $U \cap P\left(\Re_{n}^{k}\right)$, $\partial \gamma$ bounds a singular $(n-k-1)$-ball.

Theorem 3.18 gives conditions under which $\sigma$-compact subsets of $P\left(\Re_{n}^{k}\right)$ are negligible: since this statement is independent of $E^{n}$, we might call it an intrinsic negligibility theorem for $P\left(\Re_{n}^{k}\right)$. We now state our best intrinsic negligibility theorem for $P\left(\Re_{n}^{k}\right)$ (compare Theorems 0 and I of [1]).

Theorem 3.20. Assume $(n, k) \neq(3,1),(4,0)$ or $(4,1)$.

(1) A closed subset of $P\left(\Re_{n}^{k}\right)$ is strongly negligible in $P\left(\Re_{n}^{k}\right)$ if and only if it is a $Z_{n-k-2}$-set in $P\left(\Re_{n}^{k}\right)$.

(2) A subset of $P\left(\Re_{n}^{k}\right)$ is strongly negligible in $P\left(\Re_{n}^{k}\right)$ if and only if it is the countable union of $Z_{n-k-2}$-sets in $P\left(\Re_{n}^{k}\right)$.

Proof. We prove (2); the proof of (1) is similar. Let $X$ be strongly negligible in $P\left(\Re_{n}^{k}\right)$. Then, since $P\left(\Re_{n}^{k}\right)-X$ is topologically complete (a $G_{\delta}$ in $\left.P\left(\Re_{n}^{k}\right)\right), X$ is an $F_{0}$-subset. Let $X=\cup_{i=1}^{\infty} X_{i}$ where each $X_{i}$ is closed in $P\left(\Re_{n}^{k}\right)$. We will show that each $X_{i}$ is a $Z_{n-k-2^{-}}$-set in $P\left(\Re_{n}^{k}\right)$. Let $U$ be a nonempty open $(n-k-1)$ connected subset of $P\left(\Re_{n}^{k}\right)$. Clearly $U-X_{i}$ is nonempty. Let $\gamma$ be a singular ball in $U$ of dimension $\leq n-k-1$ such that $\partial \gamma \cap X_{i}=\varnothing$. Let $V$ be open in $P\left(\Re_{n}^{k}\right)$ such that $\partial \gamma \cap V=\varnothing$ and $\gamma \cap X_{i} \subset V$. Since $X$ is strongly negligible, there is a homeomorphism $h$ of $P\left(\Re_{n}^{k}\right)$ fixing $P\left(\Re_{n}^{k}\right)-V$ such that $h(V)$ $=V-X \subset V-X_{i}$. Then $h(\gamma)$ is a singular ball in $U-X$ bounded by $\partial \gamma$ and hence $U-X_{i}$ is $(n-k-2)$-connected.

Conversely, let $X=\cup_{i=1}^{\infty} X_{i}$ where each $X_{i}$ is a $Z_{n-k-2^{-}}$set in $P\left(\pi_{n}^{k}\right)$. Let $X_{i}^{\prime}$ be the closure of $X_{i}$ in $E^{n}$. By 3.1(5) and 3.17, it is enough to show that each $X_{i}^{\prime}$ is a $Z_{n-k-2^{-}}$set in $E^{n}$. Let $U$ be open in $E^{n}$. If $U$ is nonempty and $(n-k-2)$ connected, then $U \cap P\left(\Re_{n}^{k}\right)$ is nonempty and $(n-k-2)$-connected by 3.19 . 
But then $\left(U \cap P\left(\Re_{n}^{k}\right)\right)-X_{i}=\left(U-X_{i}^{\prime}\right) \cap P\left(\Re_{n}^{k}\right)$ is nonempty and $(n-k$ - 2)-connected and hence so is $U-X_{i}$, again by 3.19 .

We now turn to the pseudo-boundary. 2.13, 3.5, 3.8 and 3.12 yield

Theorem 3.21. Let $n \geq 2 k+1$ and let $X$ be a strong $Z_{n-k-2}$-set in $E^{n}$. Then $X \cap B\left(\Re_{n}^{k}\right)$ is strongly negligible in $B\left(\Re_{n}^{k}\right)$.

By 2.16, we have

Theorem 3.22. Let $n \geqslant 2 k+1$. If $X$ is a closed subset of $E^{n}$ lying in $B\left(\Re_{n}^{k}\right)$ then $X$ is strongly negligible in $B\left(\Re_{n}^{k}\right)$. In particular, any compact subset of $B\left(\Re_{n}^{k}\right)$ is strongly negligible in $B\left(\Re_{n}^{k}\right)$.

Lemma 3.23. If $n \leq 2 k+1$ and $U$ is open in $E^{n}$, then $U$ is nonempty and $(n-k-2)$-connected if and only if $U \cap B\left(\Re_{n}^{k}\right)$ is nonempty and $(n-k-2)$ connected.

Proof. If $U$ is $(n-k-2)$-connected and $\partial \gamma$ is a singular $(n-k-2)$-sphere in $U \cap B\left(\Re_{n}^{k}\right)$, let $\partial \gamma$ bound a singular $(n-k-1)$-ball $\gamma$ in $U$. Since $n-k-1$ $\leq k$, we may assume that $\gamma$ is a strong $Z_{n-k-2^{2}}$-set in $E^{n}$ (use Lemma 4.2 of [20] to make $\gamma-\partial \gamma$ "polyhedral" and then use 3.9 and general position). But now $\gamma$ can be absorbed into $U \cap B\left(\Re_{n}^{k}\right)$ keeping $\partial \gamma$ fixed and hence $U \cap B\left(\Re_{n}^{k}\right)$ is $(n-k-2)$-connected.

Suppose now that $U \cap B\left(\Re_{n}^{k}\right)$ is $(n-k-2)$-connected and let $\partial \gamma$ be a PL singular $(n-k-2)$-sphere in $U$. Then $\partial \gamma$ is a strong $Z_{n-k-2}$-set and hence can be absorbed into $U \cap B\left(\pi_{n}^{k}\right)$. But then $\partial \gamma$ bounds a singular $(n-k-1)$-ball $\gamma$ in $U \cap B\left(\pi_{n}^{k}\right)$ and hence in $U$.

Again, we would like an intrinsic negligibility theorem for $B\left(\Re_{n}^{k}\right)$. We only know how to characterize the strongly negligible closed sets in $B\left(\Re_{2 k+1}^{k}\right), k \neq 1$.

Theorem 3.24. $A$ closed subset of $B\left(\Re_{2 k+1}^{k}\right), k \neq 1$, is strongly negligible in $B\left(\Re_{2 k+1}^{k}\right)$ if and only if it is a $Z_{k-1}$-set in $B\left(\Re_{2 k+1}^{k}\right)$.

Proof. If $X$ is strongly negligible in $B\left(\pi_{2 k+1}^{k}\right)$, and $U$ is open in $B\left(\Re_{2 k+1}^{k}\right)$, then $U$ and $U-X$ are homeomorphic. Conversely, let $X$ be a $Z_{k-1}$-set in $B\left(\Re_{2 k+1}^{k}\right)$ and let $X^{\prime}$ be the closure of $X$ in $E^{n}$. By 3.1(5) and 3.21, it is enough to show that $X^{\prime}$ is a $Z_{k-1}$-set in $E^{2 k+1}$. Let $U$ be open in $E^{n}$. If $U$ is nonempty and $(k-1)-$ connected, then $B\left(\Re_{2 k+1}^{k}\right) \cap U$ is nonempty and $(k-1)$-connected by 3.23 . Then $\left(U \cap B\left(\Re_{2 k+1}^{k}\right)\right)-X=\left(U-X^{\prime}\right) \cap B\left(\Re_{2 k+1}^{k}\right)$ is nonempty and $(k-1)$ connected, and hence so is $U-X^{\prime}$, again by 3.23 .

Proof of Theorem 1.1. Set $B^{k}=B\left(\Re_{n}^{k}\right)$ and $P^{n-k-1}=P\left(\Re_{n}^{k}\right)$.

Proof of Theorem 1.2. We need only show that any $k$-dimensional compactum $X$ can be embedded in $P\left(\Re_{2 k+1}^{k}\right)$. To prove this, embed $X$ into $E^{2 k+1}$ as a strong $Z_{k-1}$-set (see 3.6 of [15]) and then, using 2.11 and 3.8, push $X$ off $B\left(\Re_{2 k+1}^{k}\right)$.

4. The polyhedral pseudo-boundaries in $E^{n}, n \neq 4$. There is a $k$-dimensional polyhedral pseudo-boundary in $E^{n}, n \neq 4$, for each integer $k,-1 \leq k<n$. It is easier to define and easier to picture than the universal pseudo-boundary, but in 
order to establish its properties we must appeal to one deep theorem: the "hauptvermutung" for $E^{n}$, now known for all $n \neq 4$ ([27], [5], [22]).

In part A we define the family $\mathcal{P}_{n}^{k}$ of tame polyhedra and verify the axioms. In part B we set up the pseudo-boundary. In part $\mathrm{C}$ we apply $\S 2$ to obtain negligibility theorems. The section ends with formal proofs of Theorems 1.3, 1.4 and 1.5: the last two seem to require a codimension 3 taming theorem.

There is a provocative relationship with the infinite-dimensional case. The usual pseudo-boundary of the Hilbert cube $I^{\omega}$ is itself the countable union of copies of $I^{\omega}$; in other words, it is the countable union of universal compacta in $I^{\omega}$. Compare with the $k$-dimensional pseudo-boundary $B\left(\Re_{n}^{k}\right)$ defined in $\S 3$ which is the countable union of universal $k$-dimensional compacta in $E^{n}$. There is also a smaller pseudo-boundary in $I^{\omega}$, defined by Anderson in [2]. This one is the countable union of finite-dimensional cubes. Compare with the polyhedral $k$ dimensional pseudo-boundary $B\left(\rho_{n}^{k}\right)$ to be defined in this section. So far a good analogy.

But more is known about the infinite-dimensional case. While the two pseudoboundaries in $I^{\omega}$ are not equivalent (in one case take $\delta$ to be the family of all $Z$ sets and in the other, take $\delta$ to be the family of all tame polyhedra or the family of all finite-dimensional $Z$-sets) the corresponding pseudo-interiors are both homeomorphic to Hilbert space $l_{2}$ (see [2]). Does the analogy with the finite-dimensional case extend this far? Letting $P\left(\Re_{n}^{k}\right)=E^{n}-B\left(\Re_{n}^{k}\right)$ and $P\left(\mathscr{P}_{n}^{k}\right)$ $=E^{n}-B\left(\mathscr{P}_{n}^{k}\right)$ we propose

Conjecture. If $n \leq 2 k+1$, then $P\left(\Re_{n}^{k}\right)$ and $P\left(\mathscr{P}_{n}^{k}\right)$ are homeomorphic(2).

A. Tame polyhedra. Once again we let $k$ and $n$ be integers, $n \geq 0$ and $-1 \leq k<n$. A (closed) subset $X$ of $E^{n}$ is a tame polyhedron if there is a homeomorphism $h$ of $E^{n}$ such that $h(X)$ is a subpolyhedron of $E^{n}$. Let $\mathcal{P}_{n}^{k}$ denote the family of all tame $k$-dimensional polyhedra in $E^{n}$.

Lemma 4.1. The family $\mathcal{P}_{n}^{k}$ satisfies Axioms 1 and 2 . If $n \geq 2 k+1$, then $\mathscr{P}_{n}^{k}$ also satisfies Axiom 3.

Proof. Axioms 1 and 2 are clearly satisfied. Moreover, Proposition 3.1 implies, except possibly when $(n, k)=(3,1),(4,0)$, or $(4,1)$, that tame $k$-dimensional polyhedra are strong $Z_{n-k-2}$-sets. If $(n, k)=(4,0)$ or $(4,1)$, then Theorem 1.1 of [17] implies that a tame $k$-dimensional polyhedron is a strong $Z_{n-k-2^{-}}$-set. The remaining case, $(n, k)=(3,1)$, is taken care of by [5]. The lemma now follows by applying $3.6,3.7$ and 3.8.

There is an easily described subset $B\left(\mathscr{P}_{n}^{k}\right)$ of $E^{n}$ which is a pseudo-boundary for $\mathscr{P}_{n}^{k}(n \neq 4)$. In the spirit of part $\mathrm{E}$ of $\S 2$, we will then be able to define a maximal family $\overline{\mathcal{P}}_{n}^{k}$ containing $\mathscr{P}_{n}^{k}$, for which $B\left(\mathscr{P}_{n}^{k}\right)$ is a pseudo-boundary. The negligibility results then follow for this family $\overline{\mathscr{P}}_{n}^{k}$.

B. The polyhedral pseudo-boundary. Throughout this section let $J_{0}$ be a rectilin-

(2) The conjecture is easily proved when $k=0$ and $n=1$. The restriction $n \leq 2 k+1$ seems reasonable: the statement is false if $k=0$ and $n \geq 2$. We thank R.D. Anderson for this observation. 
ear PL triangulation of $E^{n}$ such that all the $n$-simplexes of $J_{0}$ have the same diameter. For each integer $i \geq 1$, let $J_{i}$ be the $i$ th barycentric subdivision of $J_{0}$ and let $J_{i}^{k}$ be the $k$-skeleton of $J_{i}$. Let $B_{i}\left(\mathscr{P}_{n}^{k}\right)=\left|J_{i}^{k}\right|$ and $B^{\prime}\left(\mathscr{P}_{n}^{k}\right)=\cup_{i=1}^{\infty} B_{i}\left(\mathscr{P}_{n}^{k}\right)$. We call $B\left(\mathscr{P}_{n}^{k}\right)$ the polyhedral $k$-dimensional pseudo-boundary in $E^{n}$ and our task is to prove

Theorem 4.2. If $n \neq 4, B\left(\mathscr{P}_{n}^{k}\right)$ is a pseudo-boundary for the family $\mathscr{P}_{n}^{k}$ in $E^{n}$.

Since each $B_{i}\left(\mathscr{P}_{n}^{k}\right)$ is a tame $k$-dimensional polyhedron in $E^{n}$, we need only show that absorption holds. The case $n=4$ is excluded because we need to know that any homeomorphism of $E^{n}$ can be approximated by a PL homeomorphism: this is obvious for $n=1$, is due to Rado [27] for $n=2$, is due to Moise for $n=3$ (see also Bing's corollary to Theorem 5 in [5]), is unknown for $n=4$, and is due to Kirby-Siebenmann for $n \geq 5$ (see pp. 1-2 of [22]). For reference we state exactly what we need:

Proposition 4.3. Let $M$ and $\tilde{M}$ be PL manifolds homeomorphic to $E^{n}, n \neq 4$, let $h: M \rightarrow \tilde{M}$ be a homeomorphism, let d be a metric on $\tilde{M}$, and let $\varepsilon: M \rightarrow \mathbf{R}^{+}$be a map. Then there is a PL homeomorphism $h^{\prime}: M \rightarrow \tilde{M}$ such that $d\left(h(x), h^{\prime}(x)\right)$ $<\varepsilon(x)$ for each $x \in M$.

The proof of Theorem 4.2 is contained in the following three lemmas.

Lemma 4.4. Let $P$ be a compact $k$-dimensional subpolyhedron of $E^{n}$, let $W$ be an open set in $E^{n}$ containing $P$, and let $\varepsilon$ be a positive real number. For any integer $i \geq 1$, there exists an integer $j \geq i$ and a homeomorphism $h$ of $E^{n}$ fixing $E^{n}-W$ such that $h\left(P \cup B_{i}\left(\mathscr{P}_{n}^{k}\right)\right) \subset B_{j}\left(\mathscr{P}_{n}^{k}\right)$ and $d(x, h(x))<\varepsilon$ for each $x \in W$.

Proof. Let $K$ be a subcomplex of $E^{n}$ such that $|K|=P$. Let $m$ be an integer, $m \geq i$, such that mesh $J_{m}<\min \left\{\varepsilon, d\left(|K|, E^{n}-W\right)\right\}$. By Lemma 1.5 of [20], there is an integer $r>0$ and an $r$ th derived subdivision $J_{m}^{(r)}$ of $J_{m}$ which subdivides $|K|$ : in fact, the proof of that lemma implies that the derived subdivision $J_{m}^{(r)}$ may be taken to agree with the barycentric subdivision $J_{m+r}$ outside $W$ since mesh $J_{m}<d\left(|K|, E^{n}-W\right)$. Let $\varphi$ be the obvious simplicial isomorphism from $J_{m}^{(r)}$ onto $J_{m+r}$ and let $h=|\varphi|$. Since mesh $J_{m}<\varepsilon, d(x, h(x))$ $<\varepsilon$ for each $x \in E^{n} ; h\left(|K| \cup\left|J_{m}^{k}\right|\right) \subset\left|J_{m+r}^{k}\right|$ and $h$ fixes $E^{n}-W$. Hence the required integer $j$ is $m+r$ and the required homeomorphism is $h$.

For any open subset $U$ of $E^{n}$ we shall assume that $U$ is endowed with the natural polyhedral structure from $E^{n}$ and that a subpolyhedron of $U$, defined with respect to this natural structure, is closed in $U$.

Lemma 4.5. Let $U$ be an open subset of $E^{n}$, let $P$ be $a k$-dimensional subpolyhedron of $U$, and let $\varepsilon$ be a positive real number. Then there exists a homeomorphism $h$ of $E^{n}$ such that $h(P) \subset B\left(\mathscr{P}_{n}^{k}\right), h \mid E^{n}-U=1$, and $d(x, h(x))$ $<\varepsilon$ for each $x \in U$.

Proof. Let $W_{1}, W_{2}, \ldots$ be a star-finite open cover of $U$ and $P_{1}, P_{2}, \ldots$ a 
collection of compact $k$-dimensional subpolyhedra of $E^{n}$ such that $P=\cup_{i=1}^{\infty} P_{i}$, $P_{i} \subset W_{i}$, and $W_{1}=\varnothing$. By Lemma 2.20, we may assume that there is a sequence $\{n(i)\}$ of positive integers such that the conclusions of Lemma 2.20 hold. By induction, we shall choose a sequence of integers $\left\{j_{i}\right\}$ and a sequence of homeomorphisms $\left\{h_{i}\right\}$ of $E^{n}$ such that

(1) $h_{i} \mid E^{n}-W_{i}=1$,

(2) $h_{i}\left(P_{i} \cup B_{j_{i-1}}\left(\mathscr{P}_{n}^{k}\right)\right) \subset B_{j_{i}}\left(\Im_{n}^{k}\right)$, and

(3) $d\left(x, h_{i}(x)\right)<\varepsilon / \max \{n(1), \ldots, n(i)\}$

for each $i \geq 1$. To start the induction, set $h_{1}=1$ and $j_{1}=1$. Assume $h_{1}, \ldots$, $h_{i-1}$ and $j_{1}, \ldots, j_{i-1}$ have been constructed and simply apply Lemma 4.4 to $W_{i}, P_{i}$, and $j_{i-1}$.

Now by Lemma 2.20, there exists a homeomorphism $h$ of $E^{n}$ such that $h$ agrees with $h_{k} \cdots h_{1}$ on $W_{i}$ for each $k \geq n(i)$. $h$ is the desired homeomorphism.

Lemma 4.6. Let $X$ be a compact $k$-dimensional tame polyhedron in $E^{n}, n \neq 4$, let $W$ be an open subset of $E^{n}$ which is homeomorphic to $E^{n}$, and let $\varepsilon$ be a positive real number. Then there exists a homeomorphism $h$ of $E^{n}$ such that $h(X \cap W)$ is a subpolyhedron of $W, h \mid E^{n}-W=1$, and $d(x, h(x))<\varepsilon$ for each $x \in W$.

Proof. Let $f$ be a homeomorphism of $E^{n}$ such that $f(X)$ is a subpolyhedron of $E^{n}$. Then $f(X \cap W)$ is a subpolyhedron of $f(W)$ where $f(W)$ inherits its PL structure as an open subset of $E^{n}$. But $f(W)$ has another PL structure imposed on it by $f$ : namely, the image under $f$ of the PL structure which $W$ inherits from $E^{n}$. Let $(f(W))^{\sim}$ be $f(W)$ with the latter PL structure.

Let $\delta_{0}>0$ be such that $d\left(f^{-1}(x), f^{-1}(y)\right)<\varepsilon$ whenever $x, y \in f(X)$ and $d(x, y)<\delta_{0}$, and let $\delta: f(W) \rightarrow \mathbf{R}^{+}$be defined by $\delta(x)=\min \left\{d\left(f^{-1}(x), E^{n}\right.\right.$ $\left.-W), \delta_{0}\right\}$ for each $x \in f(W)$. Now apply Proposition 4.3 to the identity map 1: $f(W) \rightarrow(f(W))^{\sim}$ to obtain a PL homeomorphism $\tilde{h}$ such that $d(\tilde{h}(x), x)$ $<\delta(x)$ for each $x \in f(W)$. The required $h$ should agree with $f^{-1} \tilde{h} f$ on $W$ and should fix $E^{n}-W$.

Proof of Theorem 4.2. By 2.22 we need only prove that, given a tame $k$ dimensional polyhedron $X$ in $E^{n}$, an open subset $U$ in $E^{n}$, and $\varepsilon>0$, there exists a homeomorphism $h: E^{n} \rightarrow E^{n}$ such that $h(X \cap U) \subset B\left(\mathscr{P}_{n}^{k}\right), h \mid E^{n}-U=1$, and $d(x, h(x))<\varepsilon$ for each $x \in U$. Using the fact that $U$ is a PL manifold, we can choose a countable star-finite open cover $W$ of $U$ each element of which is homeomorphic to $E^{n}$ and contained in $U$. Apply Lemma 2.20 to $v$ to obtain an ordering $W_{1}, W_{2}, \ldots$ of $\vartheta$ and a sequence of integers $\{n(i)\}$ satisfying, the conclusions of Lemma 2.20. By combining Lemmas 4.5 and 4.6, there is a sequence $\left\{h_{i}\right\}$ of homeomorphisms of $E^{n}$ such that

(1) $d\left(x, h_{i}(x)\right)<\varepsilon / \max \{n(1), \ldots, n(i)\}$ for each $x \in W_{i}$,

(2) $h_{i} \mid E^{n}-W_{i}=1$, and

(3) $h_{i+1}\left(h_{i}(X) \cap W_{i+1}\right) \subset B\left(\odot_{n}^{k}\right)$

for each $i \geq 1$. Then the homeomorphism $h$ guaranteed by Lemma 2.21 has the desired properties. 
C. Negligibility results. As with the universal pseudo-boundary, we merely collect the negligibility results of $\$ 2$ and apply them to the polyhedral pseudoboundary. We note here that the maximal family $\overline{\mathscr{P}}_{n}^{k}$ in this case is not the same as $\mathscr{P}_{n}^{k}$. The Artin-Fox arc (see p. 177 of [19]), which is wild in $E^{3}$, can be absorbed into $B\left(\mathscr{P}_{3}^{1}\right)$ and the Alexander horned sphere (see p. 176 of [19]), also wild in $E^{3}$, can be absorbed into $B\left(\mathscr{P}_{3}^{2}\right)$. Let $P\left(\mathscr{P}_{n}^{k}\right)=E^{n}-B\left(\mathscr{P}_{n}^{k}\right) .2 .8,2.13,2.15,4.1$ and 4.2 yield the following two theorems:

Theorem 4.7. Let $X$ be the countable union of closed subsets of elements of $\overline{\mathscr{S}}_{n}^{k}$, $n \neq 4$. Then $X \cap P\left(\mathscr{P}_{n}^{k}\right)$ is strongly negligible in $P\left(\mathscr{P}_{n}^{k}\right)$.

Theorem 4.8. Let $n \geq 2 k+1$ and let $X$ be the finite union of closed subsets of elements of $\overline{\mathcal{P}}_{n}^{k}, n \neq 4$. Then $X \cap B\left(\mathscr{P}_{n}^{k}\right)$ is strongly negligible in $B\left(\mathscr{P}_{n}^{k}\right)$.

By 2.16 , we have

Theorem 4.9. Let $n \geq 2 k+1, n \neq 4$. Then any closed subset of $E^{n}$ lying in $B\left(\mathscr{P}_{n}^{k}\right)$ is strongly negligible in $B\left(\mathscr{P}_{n}^{k}\right)$. In particular, any compact subset of $B\left(\mathscr{P}_{n}^{k}\right)$ is strongly negligible in $B\left(\mathscr{P}_{n}^{k}\right)$.

Proof of Theorem 1.3. Any $(n-k-1)$-dimensional subpolyhedron of $E^{n}$ can be pushed successively off the $B_{i}\left(\mathscr{P}_{n}^{k}\right)$ and hence into $P\left(\mathscr{P}_{n}^{k}\right)$ (see the proof of 3.10); this proves (1). Any subset of $P\left(\mathscr{P}_{n}^{k}\right)$ has dimension at most $n-k-1$ $\leq k$, so (2) holds. (3) follows from 4.9.

Proof of Theorem 1.4. Let $X=\cup_{i=1}^{\infty} X_{i}$ where each $X_{i}$ is a compact topological polyhedron in $P\left(\odot_{n}^{k}\right)$. If $k \geq 2$, then each $X_{i}$ is a $Z_{1}$-set in $E^{n}$ (absorb a 2-ball into $\left.B\left(\mathscr{P}_{n}^{k}\right)\right)$. By a recent codimension three taming theorem due to several authors (see for example Theorem 4 of [11] and Theorem 1 of [28]) each $X_{i}$ is tame in $E^{n}$. If $k=0$ then each $X_{i}$ is obviously tame in $E^{n}$. Now apply 4.7.

Proof of Theorem 1.5. (1) follows from 1.4 when $k \geq 2$ and is elementary when $k=0$; (2) comes from 1.3(3); and (3) comes from 1.3 and 4.2.

5. Pseudo-boundaries in topological manifolds. In this section we indicate briefly how the notions introduced in $\$ 3$ and $\$ 4$ can be extended to manifolds.

A topological n-manifold is a separable metrizable space each point of which has a neighborhood homeomorphic to $E^{n}$. Throughout this section $M$ will denote a fixed topological $n$-manifold. A euclidean chart for $M$ is a pair $(h, W)$ where $W$ is an open subset of $M$ and $h: E^{n} \rightarrow W$ is a homeomorphism.

A. The universal pseudo-boundaries. Let $k$ and $n$ be integers, $-1 \leq k<n$. A closed subset $X$ of $M$ is a local strong $Z_{n-k-2}$-set in $M$ if for each point $x$ in $X$ there exists a euclidean chart $(h, W)$ such that $x \in W$ and $h^{-1}(X)$ is a strong $Z_{n-k-2^{-}}$-set in $E^{n}$. Using the hauptvermutung for $E^{n}$ (Proposition 4.3) when $n \neq 4$ and a dimension argument when $n=4$ it is easy to show that a closed subset $X$ of $E^{n}$ is a local strong $Z_{n-k-2}$-set in $E^{n},(n, k) \neq(4,0)$ or $(4,1)$, if and only if it is a strong $Z_{n-k-2}$-set in $E^{n}$. This implies

Lemma 5.1. Let $(n, k) \neq(4,0)$ or $(4,1)$. $A$ closed subset $X$ in $M$ is a local strong 
$Z_{n-k-2}$-set in $M$ if and only if for each euclidean chart $(h, W)$ in $M, h^{-1}(X)$ is a strong $Z_{n-k-2}$-set in $E^{n}$.

Let $\Re_{M}^{k}$ denote the family of all local strong $Z_{n-k-2}$-sets in $M$.

Lemma 5.2. $\Re_{M}^{k}$ satisfies Axioms 1 and 2. If $n \geq 2 k+1$ and $(n, k) \neq(4,0)$ or $(4,1)$, then $\Re_{M}^{k}$ also satisfies Axiom 3.

Proof. Axioms 1 and 2 are clearly satisfied. To prove Axiom 3, let $X$ be a finite union of closed subsets of elements of $\pi_{M}^{k}$. By 3.6, 3.7, and 5.1, $h^{-1}(X)$ is a strong $Z_{n-k-2}$-set in $E^{n}$ for any euclidean chart $(h, W)$. Using charts to cover $X$, we can push $X$ off itself inside each of these charts and then use an argument similar to the proof of 2.11 to show that $X$ is thin in $M$.

We now construct a pseudo-boundary for $\pi_{M}^{k}$ in $M$. Let $\left\{\left(h_{i}, W_{i}\right)\right\}$ be a countable collection of charts of $M$ such that $\left\{W_{i}\right\}$ covers $M$. Let $B\left(\Re_{M}^{k}\right)$ $=\cup_{i=1}^{\infty} h_{i}\left(B\left(\Re_{n}^{k}\right)\right)$. We shall show that $B\left(\Re_{M}^{k}\right)$ is a pseudo-boundary and hence, by Theorem 2.5 , that the construction is essentially independent of the particular charts $\left(h_{i}, W_{i}\right)$. Hence we are justified in calling $B\left(\Re_{M}^{k}\right)$ the universal $k$-dimensional pseudo-boundary of $M$.

Theorem 5.3. If $(n, k) \neq(4,0)$ or $(4,1)$, then $B\left(\Re_{M}^{k}\right)$ is a pseudo-boundary for $\mathfrak{T}_{M}^{k}$ in $M$.

Proof. It is clear that $B\left(\Re_{M}^{k}\right)$ is the countable union of elements of $\mathfrak{T}_{M}^{k}$. To prove absorption, let $U$ be open in $M$, let $\varepsilon>0$, and let $X \in \mathfrak{T}_{M}^{k}$. Then $\left\{W_{i} \cap U\right\}$ is a covering of the separable metric space $U$ and hence there is a sequence of triples $\left\{\left(f_{i}, U_{i}, U_{i}^{\prime}\right)\right\}$ where each $U_{i}^{\prime}$ is open in $E^{n} ; f_{i}$ is a homeomorphism of $U_{i}^{\prime}$ onto the open subset $U_{i}$ of $U$; each $f_{i}$ agrees with some $h_{j}$ where $h_{j}$ is a homeomorphism in one of the charts $\left(h_{j}, W_{j}\right)$ used to construct $B\left(\Re_{M}^{k}\right)$; and the collection $\left\{U_{i}\right\}$ covers $U$ and is a star-finite refinement of the cover $\left\{W_{i} \cap U\right\}$ of $U$. Now fix an integer $i$ and consider $f_{i}^{-1}\left(X \cap U_{i}\right)$. Then $f_{i}^{-1}\left(X \cap U_{i}\right)$ can be absorbed into $B\left(\Re_{n}^{k}\right)$ and hence $X \cap U_{i}$ can be absorbed into $B\left(\Re_{M}^{k}\right)$ without moving points outside $U_{i}$. Now since the cover $\left\{U_{i}\right\}$ is a star-finite cover, the theorem follows from Lemma 2.20. We leave the epsilonics to the reader.

The usual uniqueness and negligibility theorems follow and we leave both their statements and proofs to the interested reader.

B. The polyhedral pseudo-boundaries. A closed subset $X$ of $M$ is a local tame $k$ dimensional polyhedral set in $M$ if for each point $x$ in $X$, there is a euclidean chart $(h, W)$ of $M$ such that $x \in W$ and $h^{-1}(X)$ is a tame $k$-dimensional polyhedron in $E^{n}$. Let $\mathscr{P}_{M}^{k}$ denote the collection of all local tame $k$-dimensional polyhedral sets in $M$. Note: we do not claim that the elements of $\mathscr{P}_{M}^{k}$ are topological polyhedra.

Lemma 5.4. $\mathscr{P}_{M}^{k}$ satisfies Axioms 1 and 2. If $n \geq 2 k+1$ and $(n, k) \neq(4,0)$ or $(4,1)$, then $\mathcal{P}_{M}^{k}$ also satisfies Axiom 3. 
Proof. 1 and 2 are trivial. A local tame $k$-dimensional polyhedral set in $M$ is obviously a local strong $Z_{n-k-2}$-set and hence 3 follows from 5.2.

Again let $\left\{\left(h_{i}, W_{i}\right)\right\}$ be a countable collection of euclidean charts of $M$ such that $\left\{W_{i}\right\}$ covers $M$ and let $B\left(\mathscr{P}_{M}^{k}\right)=\cup_{i=1}^{\infty} h_{i}\left(B\left(\mathscr{P}_{n}^{k}\right)\right)$ : it is the polyhedral $k$-dimensional pseudo-boundary of $M$. Again, 2.5 implies that it is essentially independent of the charts $\left\{\left(h_{i}, W_{i}\right)\right\}$.

Theorem 5.4. If $n \neq 4$, then $B\left(\mathscr{P}_{M}^{k}\right)$ is a pseudo-boundary for $\mathscr{P}_{M}^{k}$ in $M$.

Proof. Use 2.20 and 4.3. The proof is not quite the same as that of 5.2 since we do not claim a lemma analogous to 5.1.

Again the uniqueness and negligibility theorems follow.

\section{REFERENCES}

1. R. D. Anderson, Strongly negligible subsets in Fréchet manifolds, Bull. Amer. Math. Soc. 75 (1969), 64-67. MR 38 \#6634.

2. - On sigma-compact subsets of infinite-dimensional spaces, Louisiana State University (preprint).

3. R. D. Anderson, D. W. Henderson and J. E. West, Negligible subsets of infinite-dimensional manifolds, Compositio Math. 21 (1969), 143-150. MR 39 \#7630.

4. C. Bessaga and A. Pełczyński, The estimated extension theorem, homogeneous collections and skeletons, and their application to topological classification of linear metric spaces and convex sets, Fund. Math. 69 (1970), 153-190. MR 42 \#8227.

5. R. H. Bing, Locally tame sets are tame, Ann. of Math. (2) 59 (1954), 145-158. MR 15, 816.

6. R. H. Bing and J. M. Kister, Taming complexes in hyperplanes, Duke Math. J. 31 (1964), 491-511. MR 29 \# 1626.

7. H. G. Bothe, Eine Einbettung $m$-dimensionaler Mengen in einen $(m+1)$-dimensionalen absoluten Retrakt, Fund. Math. 52 (1963), 209-224. MR 27 \# 1953.

8.—, Ein eindimensionales Kompaktum im $E^{3}$, das sich nicht lagetreu in die Mengersche Universalkurve einbetten lässt, Fund. Math 54 (1964), 251-258. MR 29 \#6470.

9. J. L. Bryant, On embeddings of compacta in Euclidean space, Proc. Amer. Math. Soc. 23 (1969), 46-51. MR 39 \#6286.

10. - On embeddings of 1-dimensional compacta in $E^{5}$, Duke Math. J. 38 (1971), 265-270. MR 43 \# 1154.

11. A. V. Cernavskiī, Topological imbeddings of manifolds, Dokl. Akad. Nauk SSSR 187 (1969), 1247-1250 = Soviet Math. Dokl. 10 (1969), 1037-1041. MR 41 \#4547.

12. T. A. Chapman, Dense sigma-compact subsets of infinite-dimensional manifolds, Trans. Amer. Math. Soc. 154 (1971), 399-426. MR 44 \# 1058.

13. - Hilbert cube manifolds, Bull. Amer. Math. Soc. 76 (1970), 1326-1330. MR 44 \#3352.

14. - On the structure of Hilbert cube manifolds, Compositio Math. 24 (1972), 329-353.

15. R. Geoghegan and R. R. Summerhill, Concerning the shapes of finite-dimensional compacta, Trans. Amer. Math. Soc. 179 (1973), 281-292.

16. - Infinite-dimensional methods in finite-dimensional geometric topology', Bull. Amer. Math. Soc. 78 (1972), 1009-1014.

17. H. Gluck, Embeddings in the trivial range, Ann. of Math. (2) 81 (1965), 195-210. MR 30 \#3456.

18. D. W. Henderson and J. E. West, Triangulated infinite-dimensional manifolds, Bull. Amer. Math. Soc. 76 (1970), 655-660. MR 41 \#2714.

19. J. G. Hocking and G. S. Young, Topology, Addison-Wesley, Reading, Mass., 1961. MR 23 \# A2857.

20. J. F. P. Hudson, Piecewise linear topology, University of Chicago Lecture Notes, prepared with 
the assistance of J. L. Shaneson and J. Lees, Benjamin, New York, 1969. MR 40 \#2094.

21. W. Hurewicz and H. Wallman, Dimension theory, Princeton Math. Series, vol. 4, Princeton Univ. Press, Princeton, N.J., 1941. MR 3, 312.

22. R. Kirby, Lectures on triangulations of manifolds, mimeographed notes, University of California, Los Angeles, Calif., 1969.

23. S. Lefschetz, On compact spaces, Ann. of Math. 32 (1931), 521-538.

24. D. R. McMillan, Taming Cantor sets in $E^{n}$, Bull. Amer. Math. Soc. 70 (1964), 706-708. MR 29 \# 1628.

25. D. R. McMillan and H. Row, Tangled embeddings of one-dimensional continua, Proc. Amer. Math. Soc. 22 (1969), 378-385. MR 39 \# 7571.

26. K. Menger, Über umfassendste n-dimensionale Mengen, Nederl. Akad. Wetensch.Proc. 29 (1926), 1125-1128.

27. T. Rado, Über den Begriff Riemannschen Fläche, Acta. Univ. Szeged. Sect. Sci. Math. 2 (1925), $101-121$.

28. T. B. Rushing, Taming codimension three embeddings, Bull. Amer. Math. Soc. 75 (1969), 815-820. MR 39 \#6328.

29. M. A. Śtan'ko, The embedding of compacta in Euclidean space, Mat. Sb. 83 (125) (1970), 234-255 = Math. USSR Sb. 12 (1970), 234-254. MR 42 \#6804.

30. - Solution of Menger's problem in the class of compacta, Dokl. Akad. Nauk. SSR 201 (1971), 1299-1302 = Soviet Math. Dokl. 12 (1971), 1846-1849.

31. H. Toruńczyk, Skeletonized sets in complete metric spaces and homeomorphisms of the Hilbert cube, Bull. Acad. Polon. Sci. Sér. Sci. Math. Astronom. Phys. 18 (1970), 119-126. MR 41 \#9194.

32. J. E. West, The ambient homeomorphy of an incomplete subspace of infinite-dimensional Hilbert spaces, Pacific J. Math. 34 (1970), 257-267. MR 43 \#2748.

33. R. Geoghegan and R. R. Summerhill, Finite-dimensional spaces having many of the topological properties of separable Hilbert spaces $l_{2}$, Bull. Acad. Polon. Sci. Sér. Sci. Math. Astronom. Phys. (to appear).

School of Mathematics, Institute for Advanced Study, Princeton, New Jersey 08540

Current address (R. Geoghegan): Department of Mathematical Sciences, State University of New York at Binghamton, Binghamton, New York 13901

Current address (R. R. Summerhill): Department of Mathematics, Kansas State University, Manhattan, Kansas 66502 